\title{
Use of Compliant Hinges to Tailor Flight Dynamics of Unmanned Aircraft
}

\author{
Emily A. Leylek* and Mark Costello \\ Georgia Institute of Technology, Atlanta, Georgia 30332
}

DOI: $\underline{10.2514 / 1 . C 033056}$

\begin{abstract}
The nexus of advanced manufacturing methods, computer-aided design tools, and modern structural-analysis software has enabled the design and fabrication of structurally complex wing structures with unique features. This is particularly true for small unmanned aircraft, in which discrete structural hinges can easily be integrated into the overall vehicle design. This paper examines the use of discrete structural hinges for tailoring the low-frequency flight dynamics of the vehicle. For sufficiently soft discrete structural hinges, substantial coupling between flexible and rigid modes occurs, leading to the potential to modify the flight dynamic behavior through structural flexibility. Using a multibody flight dynamics simulation tool with a nonlinear lifting-line aerodynamic representation, different structural-hinge elastic properties, orientation, and location on the aircraft are examined. The results for a small unmanned aircraft indicate that flexibility mostly affects the longitudinal modes and associated handling qualities of the vehicle. Changes in the short-period and phugoid modes due to flexibility caused by a set of discrete structural hinges are often antagonistic. A structural-hinge stiffness above a certain critical value tends to improve short-period flying qualities; below this value, the short-period flying qualities degrade. In addition to the stiffness characteristics of the discrete structural hinge, orientation of the hinge has a significant effect on flight dynamics. Orienting the structural hinge to produce pitch-flap coupling can triple the short-period-mode damping, which could improve a nominally Level 3 rated aircraft to a Level 1 rated aircraft.
\end{abstract}

\begin{tabular}{|c|c|c|}
\hline \multicolumn{3}{|r|}{ Nomenclature } \\
\hline $\bar{\alpha}_{P / Q}$ & $=$ & $\begin{array}{l}\text { angular acceleration of body } P \text { with respect to } \\
\text { reference frame } Q, \mathrm{rad} / \mathrm{s}^{2}\end{array}$ \\
\hline$\oplus_{B} / I$ & $=$ & $\begin{array}{l}\text { acceleration of center of gravity of body } B \text { with } \\
\text { respect to inertial reference frame, } \mathrm{m} / \mathrm{s}^{2}\end{array}$ \\
\hline $\mathbb{C}_{B}$ & $=$ & $\begin{array}{l}\text { component operator in generic reference } \\
\text { frame } B\end{array}$ \\
\hline & $=$ & lift coefficient, drag coefficient [nd] \\
\hline$e_{l}$ & & $\begin{array}{l}\text { roll, pitch, yaw aerodynamic moment co- } \\
\text { efficients in fuselage body reference frame } \\
\text { [nd] }\end{array}$ \\
\hline$C_{L_{0}}, C_{D_{0}}$ & $=$ & $\begin{array}{l}\text { lift coefficient, drag coefficient at zero angle of } \\
\text { attack [nd] }\end{array}$ \\
\hline$C_{L_{s}}, C_{Y_{s}}, C_{D_{s}}$ & $=$ & $\begin{array}{l}\text { lift, side force, drag aerodynamic force } \\
\text { coefficient derivatives with respect to variable } \\
s \text { [nd] }\end{array}$ \\
\hline$C_{l_{s}}, C_{m_{s}}, C_{n_{s}}$ & $=$ & $\begin{array}{l}\text { roll, pitch, yaw aerodynamic moment co- } \\
\text { efficient derivatives with respect to variable } \\
s \text { [nd] }\end{array}$ \\
\hline$C_{X}, C_{Y}, C_{Z}$ & $=$ & $\begin{array}{l}\text { aerodynamic force coefficients in the fuselage } \\
\text { body reference frame [nd] }\end{array}$ \\
\hline$F_{\mathrm{RH}}$ & $=$ & left-hinge, right-hinge joint constraint forces, $\mathrm{N}$ \\
\hline & $=$ & acceleration due to gravity, $\mathrm{m} / \mathrm{s}^{2}$ \\
\hline $\bar{H}_{B}^{\oplus}$ & $=$ & $\begin{array}{l}\text { angular momentum about center of gravity of } \\
\text { body } B \text { with respect to inertial reference frame, } \\
\mathrm{kg} \cdot \mathrm{m}^{2} / \mathrm{s}\end{array}$ \\
\hline & - & mass moment of inertia matrix, $\mathrm{kg} \cdot \mathrm{m}^{2}$ \\
\hline${ }_{F}, \overline{\boldsymbol{J}}_{F}, \underline{\boldsymbol{k}}$ & & $\begin{array}{l}\text { ctors for fuselage body reference frame } \\
\text { ctors for inertial reference frame }\end{array}$ \\
\hline
\end{tabular}

Received 30 June 2014; revision received 20 December 2014; accepted for publication 20 December 2014; published online 20 April 2015. Copyright $\odot$ 2014 by the American Institute of Aeronautics and Astronautics, Inc. All rights reserved. Copies of this paper may be made for personal or internal use, on condition that the copier pay the $\$ 10.00$ per-copy fee to the Copyright Clearance Center, Inc., 222 Rosewood Drive, Danvers, MA 01923; include the code 1533-3868/15 and $\$ 10.00$ in correspondence with the CCC.

*Graduate Student, Guggenheim School of Aerospace Engineering. Student Member AIAA.

${ }^{\dagger}$ David S. Lewis Professor of Autonomy, Guggenheim School of Aerospace Engineering, Woodruff School of Mechanical Engineering. Associate Fellow AIAA.

\begin{tabular}{|c|c|c|}
\hline$\overline{\boldsymbol{I}}_{L}, \overline{\boldsymbol{J}}_{L}, \overline{\boldsymbol{K}}_{L}$ & $=$ & $\begin{array}{l}\text { basis vectors for left-wing body reference } \\
\text { frame }\end{array}$ \\
\hline$\overline{\boldsymbol{I}}_{\mathrm{LF}}, \overline{\boldsymbol{J}}_{\mathrm{LF}}, \overline{\boldsymbol{K}}_{\mathrm{LF}}$ & $=$ & $\begin{array}{l}\text { basis vectors for left-fuselage-hinge joint } \\
\text { reference frame }\end{array}$ \\
\hline$\overline{\boldsymbol{I}}_{\mathrm{LH}}, \overline{\boldsymbol{J}}_{\mathrm{LH}}, \overline{\boldsymbol{K}}_{\mathrm{LH}}$ & $=$ & $\begin{array}{l}\text { basis vectors for left-hinge-wing joint } \\
\text { reference frame }\end{array}$ \\
\hline$M_{\mathrm{LH}}, M_{\mathrm{RH}}$ & $=$ & $\begin{array}{l}\text { left-hinge, right-hinge joint constraint mo- } \\
\text { ments, } \mathrm{N} \cdot \mathrm{m}\end{array}$ \\
\hline$m$ & $=$ & mass, $\mathrm{kg}$ \\
\hline$p, q, r$ & $=$ & $\begin{array}{l}\text { angular-velocity measure numbers in body } \\
\text { reference frame, } \mathrm{rad} / \mathrm{s}\end{array}$ \\
\hline$Q_{d} \bar{P} / d t$ & $=$ & $\begin{array}{l}\text { ve of vector } \boldsymbol{P} \text { with respect to the } \\
\text { frame }\end{array}$ \\
\hline $\bar{r}_{\mathrm{LH} \rightarrow \oplus_{L}}$ & $=$ & $\begin{array}{l}\text { tor from left-hinge position to left- } \\
\text { of gravity, } m\end{array}$ \\
\hline$\overline{\boldsymbol{r}}_{O \rightarrow \oplus_{F}}$ & $=$ & $\begin{array}{l}\text { in of inertial reference } \\
\text { y of fuselage, } m\end{array}$ \\
\hline$\overline{\boldsymbol{r}}_{\oplus_{F} \rightarrow \mathrm{LH}}$ & $=$ & $\begin{array}{l}\text { om center of gravity of } \\
\text { ge position, } m\end{array}$ \\
\hline $\mathbb{S}_{B}$ & $=$ & $\begin{array}{l}\text { skew symmetric cross-product operator in } \\
\text { generic reference frame } B\end{array}$ \\
\hline$S_{\omega}$ & $=$ & $\begin{array}{l}\text { ric cross-product operator acting } \\
\text { es }\end{array}$ \\
\hline$u, v, w$ & $=$ & $\begin{array}{l}\text { velocity-vector measure numbers in body } \\
\text { reference frame, } \mathrm{m} / \mathrm{s}\end{array}$ \\
\hline$u_{i}, v$ & $=$ & components of induced velocity vector, $\mathrm{m} / \mathrm{s}$ \\
\hline & $=$ & components of freestream velocity vector, $\mathrm{m} / \mathrm{s}$ \\
\hline $\bar{V}_{\oplus_{B} / I}$ & $=$ & $\begin{array}{l}\text { f center of gravity of body } B \text { with } \\
\text { inertial reference frame, } \mathrm{m} / \mathrm{s}\end{array}$ \\
\hline $\bar{V}_{\infty}, V_{\infty}$ & $=$ & $\begin{array}{l}\text { am velocity vector, magnitude of } \\
\text { am velocity vector, } \mathrm{m} / \mathrm{s}\end{array}$ \\
\hline$x, y, z$ & $=$ & $\begin{array}{l}\text {-vector measure numbers in inertial } \\
\text { e frame, } m\end{array}$ \\
\hline$\alpha, \beta$ & $=$ & ack, sideslip angle, rad \\
\hline$\beta_{L}, \beta_{R}$ & $=$ & $\begin{array}{l}\text { sht-wing flap angle about the } \\
\text { d }\end{array}$ \\
\hline 2 & & dih \\
\hline$\Gamma_{p}$ & $=$ & $\begin{array}{l}\text { circulation strength of panel horseshoe vortex, } \\
\mathrm{m}^{2} / \mathrm{s}\end{array}$ \\
\hline$\delta_{\mathrm{LE}}, \delta_{\mathrm{RE}}, \delta_{R}$ & $=$ & $\begin{array}{l}\text { left-elevon deflection, right-elevon deflection, } \\
\text { rudder deflection, rad }\end{array}$ \\
\hline $\bar{\varepsilon}$ & & downwash, or induced velocity, vector, $\mathrm{m} / \mathrm{s}$ \\
\hline & & 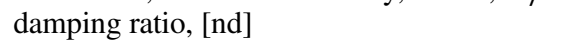 \\
\hline
\end{tabular}

$\overline{\boldsymbol{I}}_{L}, \overline{\boldsymbol{J}}_{L}, \overline{\boldsymbol{K}}_{L} \quad=$ basis vectors for left-wing body reference $\overline{\boldsymbol{I}}_{\mathrm{LF}}, \overline{\boldsymbol{J}}_{\mathrm{LF}}, \overline{\boldsymbol{K}}_{\mathrm{LF}}=$ basis vectors for left-fuselage-hinge joint reference frame reference frame ments, $\mathrm{N} \cdot \mathrm{m}$

mass, $\mathrm{kg}$ reference frame, $\mathrm{rad} / \mathrm{s}$

$Q$ reference frame

position vector from left-hinge position to leftwing center of gravity, $\mathrm{m}$ frame to center of gravity of fuselage, $m$ fuselage to left-hinge position, $\mathrm{m}$

skew symmetric cross-product operator in generic reference frame $B$ on angular rates

reference frame, $\mathrm{m} / \mathrm{s}$

components of induced velocity vector, $\mathrm{m} / \mathrm{s}$

$=$ components of freestream velocity vector, $\mathrm{m} / \mathrm{s}$

velocity of center of gravity of body $B$ with respect to inertial reference frame, $\mathrm{m} / \mathrm{s}$

freestream velocity vector, $\mathrm{m} / \mathrm{s}$

position-vector measure numbers in inertial

angle of attack, sideslip angle, rad hinge axis, rad

$=$ dihedral angle, rad

circulation strength of panel horseshoe vortex, $\mathrm{m}^{2} / \mathrm{s}$ rudder deflection, rad

$=$ damping ratio, $[\mathrm{nd}]$ 


$\begin{array}{lll}\rho_{\infty} & = & \text { freestream density, } \mathrm{kg} / \mathrm{m}^{3} \\ \phi, \theta, \psi & = & \text { Euler angle rotation parameters, rad } \\ \omega_{n} & = & \text { natural frequency, rad } / \mathrm{s} \\ \bar{\omega}_{P / Q} & =\begin{array}{l}\text { angular velocity of body } P \text { with respect to } \\ \end{array} & \text { reference frame } Q, \mathrm{rad} / \mathrm{s}\end{array}$

Subscripts

$\begin{array}{lll}F & = & \text { fuselage body } \\ L & & \text { left-wing body } \\ R & = & \text { right-wing body } \\ \oplus & = & \text { center of gravity }\end{array}$

\section{Introduction}

A DVANCES in manufacturing methods now allow complex multimaterial structures to be constructed cost effectively [1-3] These new manufacturing techniques make it possible to create discrete joints of compliant material with particular desirable stiffness and damping characteristics incorporated into a larger, rigid structure. This is especially practical in the fabrication of micro- and small-unmanned-aircraft systems [4-6]. Introducing flexibility into the wing of a small unmanned aircraft via a chordwise discrete revolute hinge can be used to alter the dynamics of the aircraft. This includes the low-frequency flight dynamic modes, such as the shortperiod, phugoid, roll, Dutch roll, and spiral modes.

A discrete structural hinge can be used as a design tool to shape the flight dynamic characteristics much like wing dihedral, twist, etc., is used. Numerous authors have considered wing flexibility in the context of flight dynamics. Passive articulation systems on aircraft have been shown to reduce gust sensitivity $[7,8]$. Porter and Brown [9] studied aircraft equipped with a free wing, which allowed pitching about a spanwise axis ahead of the aerodynamic center of the wing. They found that such a wing reduced disturbances from gusts and improved handling qualities [9]. Birds and insects are known to exploit the compliancy in their joints to achieve desirable wing motion, which reduces energy consumption [2, 10,11]. Krus [11] showed how flexible spanwise hinges or chordwise compliant joints with swept joint axes stabilize tailless birdlike aircraft. Rotorcraft have long used such a hinge, denoted as a $\delta_{3}$ hinge, for pitch-flap coupling of blades to reduce the pitch of the blade as it flaps [12]. As far back as 1928, Waterman [13] proposed an aircraft with hinged wings with $\delta_{3}$ orientations that would reduce the incidence angle as the wing flapped due to wind gusts and heavy turbulence. The wings were connected to the fuselage by a pneumatic system that would act both as a shock absorber and as a means to set the configuration of the wings for different phases of flight [13]. Aeroelastic tailoring uses the plies in composite structures to angle the bending axis forward or aft, similar to $\delta_{3}$ orientations, to introduce cross-coupling between bending and twisting of the structure. This produces wash-in or washout on the wing, which can be used to passively control flutter onset, divergence, induced drag, lift effectiveness, control effectiveness, and/or maneuver load relief $[14,15]$. Pitt conducted static and dynamic aeroelastic design studies using a physical, elastic $\delta_{3}$ hinge of various orientations and stiffnesses to create wash-in and wash-out that affected flutter characteristics and divergence speeds similar to aeroelastic tailoring with composites [16].

Ameri et al. conducted a study of the dynamic response of a flying wing with articulated winglets and showed how dynamic modes change as the vehicle is trimmed through varying symmetric dihedral angles for two winglet sizes [17]. A series of papers by Abdulrahim and Lind [18-20] investigated symmetric variable gull-wing morphing in simulation and flight tests. The variable gull wing is used as a slow control effector to deform between flight modes and to change the glide ratio, climb performance, and stall characteristics. The effect of different gull angles on the dynamic modes of the vehicle is also shown [18-20]. A gull-wing aircraft with a hinge to control the outboard wing sweep used the change in wing sweep to vary the static margin and longitudinal handling qualities [21]. Paranjape et al. used bifurcation analysis to investigate symmetric and asymmetric dihedral on an articulated wing aircraft, and the effect on stability [22].

In a subsequent paper, Paranjape et al. extended this work to an articulated aircraft with flexible wings and found that moderately flexible wings with a Young's modulus on the order of magnitude of 10 and higher did not substantially affect the stability of the vehicle in coordinated turning flight, although highly flexible wings could improve turning performance [23]. Babcock and Lind [24] studied how changes in bending stiffness and torsional stiffness of a flexible wing on a micro air vehicle affect the rigid-body modes. They discovered that the torsional stiffness had little effect on rigid-body modes, but the bending stiffness caused large changes in dihedral and the state of the vehicle in trim, significantly affecting the lateral modes of the vehicle [24]. Numerous other studies have investigated the coupling between rigid-body modes and structural modes brought on by flexible wings and methods for approximating the changes in rigid-body dynamics from this coupling [25-31]. Several studies tie these changes in the rigid modes from flexibility to changes in the handling qualities, showing that highly elastic airframes have degraded flying qualities compared to their rigid counterparts [32-34].

This paper builds on the previous work on the stability of articulated wing aircraft and flexible aircraft. Unlike the current literature on flexible aircraft wherein the entire wing is assumed to be elastic, this work focuses on wing articulation provided by a discrete compliant hinge formed by new multimaterial fabrication processes. Changes in traditional rigid-body dynamic modes of the vehicle are examined as a function of stiffness and damping of discrete structural hinges. Rather than allowing the wings to displace to their natural equilibrium dihedral in different flight conditions, the dihedral angle is forced to be equivalent in all cases to isolate the effects of hinge properties on the flight dynamics of the vehicle. The effect of $\delta_{3}$ hinge orientations (pitch-flap coupling) is extended to fixed-wing aircraft stability analysis, and a $\delta_{2}$ hinge orientation (sweep-flap coupling) is also introduced. The hinge location, either where it connects the root of the wing to the fuselage or its location spanwise on the wing, is also considered. The sensitivity of flight dynamic modes to changes in the elastic configuration is examined via static aeroelastic analysis. Also, changes in the dynamics of the vehicle are correlated to handlingqualities ratings to show how the design parameters of a flexible hinge can be used to tailor the flying qualities of the vehicle. Example results are shown for a nominal small unmanned aircraft.

\section{Simulation Methodology}

A compliant hinge embedded in a wing structure through multimaterial fabrication permits the wing to articulate relative to the fuselage through large, time-varying angles. As such, prediction of the performance of this physical mechanism requires a multibody simulation to incorporate the dynamics of this relative motion.

\section{A. Aircraft Geometry}

Each wing is attached to the fuselage with a revolute joint, adding one degree of freedom (DOF) per wing to the standard six-DOF rigidbody aircraft model. The system is composed of three bodies (fuselage, left wing, and right wing) with a total of eight DOF. Each body has a body reference frame with the origin located at the body center of gravity (c.g.). A frame aligned with the joint is also defined at the joint on each body. A schematic of the dynamic system is depicted in Fig. 1. The transformation between the inertial frame and the body frame of the fuselage is given in Eq. (1). Euler angles are used for this transformation matrix, Eq. (2). Equations (3-5) define the rotations between the body frames of the system. The rotation matrix $T_{\beta_{L}}$ is the rotation through the flap angle, $\beta_{L}$, of the wing relative to the fuselage about the joint axis, given in Eq. (ㅇ).

$$
\left\{\begin{array}{c}
\overline{\boldsymbol{I}}_{F} \\
\overline{\boldsymbol{J}}_{F} \\
\overline{\boldsymbol{K}}_{F}
\end{array}\right\}=\left[T_{F I}\right]\left\{\begin{array}{c}
\overline{\boldsymbol{I}}_{I} \\
\overline{\boldsymbol{J}}_{I} \\
\overline{\boldsymbol{K}}_{I}
\end{array}\right\}
$$




$$
\left[T_{F I}\right]=\left[\begin{array}{ccc}
\cos \theta \cos \psi & \cos \theta \sin \psi & -\sin \theta \\
\sin \phi \sin \theta \cos \psi-\cos \phi \sin \psi & \sin \phi \sin \theta \sin \psi+\cos \phi \cos \psi & \sin \phi \cos \theta \\
\cos \phi \sin \theta \cos \psi+\sin \phi \sin \psi & \cos \phi \sin \theta \sin \psi-\sin \phi \cos \psi & \cos \phi \cos \theta
\end{array}\right]
$$

$$
\begin{gathered}
\left\{\begin{array}{c}
\overline{\boldsymbol{I}}_{\mathrm{LF}} \\
\overline{\boldsymbol{J}}_{\mathrm{LF}} \\
\overline{\boldsymbol{K}}_{\mathrm{LF}}
\end{array}\right\}=\left[T_{\mathrm{LF}}\right]\left\{\begin{array}{c}
\overline{\boldsymbol{I}}_{F} \\
\overline{\boldsymbol{J}}_{F} \\
\overline{\boldsymbol{K}}_{F}
\end{array}\right\} \\
\left\{\begin{array}{c}
\overline{\boldsymbol{I}}_{\mathrm{LH}} \\
\overline{\boldsymbol{J}}_{\mathrm{LH}} \\
\overline{\boldsymbol{K}}_{\mathrm{LH}}
\end{array}\right\}=\left[T_{\beta_{L}}\right]\left\{\begin{array}{c}
\overline{\boldsymbol{I}}_{\mathrm{LF}} \\
\overline{\boldsymbol{J}}_{\mathrm{LF}} \\
\overline{\boldsymbol{K}}_{\mathrm{LF}}
\end{array}\right\} \\
\left\{\begin{array}{c}
\overline{\boldsymbol{I}}_{L} \\
\overline{\boldsymbol{J}}_{L} \\
\overline{\boldsymbol{K}}_{L}
\end{array}\right\}=\left[T_{\mathrm{LH}}\right]^{T}\left\{\begin{array}{c}
\overline{\boldsymbol{I}}_{\mathrm{LH}} \\
\overline{\boldsymbol{J}}_{\mathrm{LH}} \\
\overline{\boldsymbol{K}}_{\mathrm{LH}}
\end{array}\right\} \\
{\left[T_{\beta_{L}}\right]=\left[\begin{array}{ccc}
1 & 0 \\
0 & \cos \left(\beta_{L}\right) & \sin \left(\beta_{L}\right) \\
0 & -\sin \left(\beta_{L}\right) & \cos \left(\beta_{L}\right)
\end{array}\right]}
\end{gathered}
$$

Equation (7) defines the complete transformation matrix, $T_{L}$, from the fuselage body frame to the left-wing body frame. Figure 2 depicts the relations between all of the reference frames and transformations between them. The rotation angles between frames are noted above the arrows connecting the frames, and the transformation matrix designations are noted below the arrows in the figure.

$$
T_{L}=T_{\mathrm{LH}}^{T} T_{\beta_{L}} T_{\mathrm{LF}}
$$

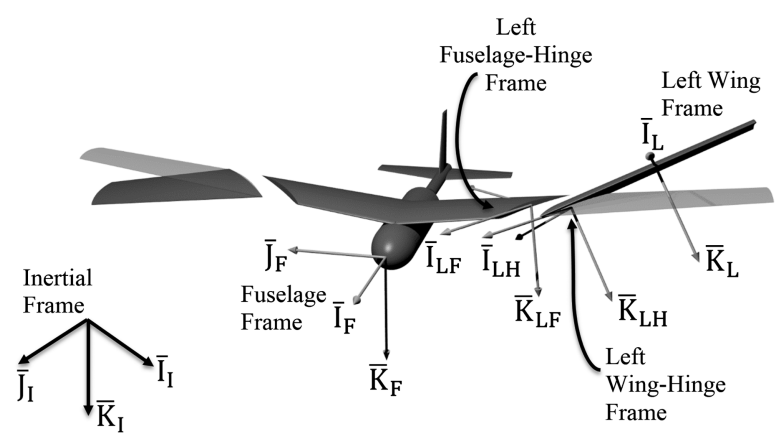

Fig. 1 Articulated wing aircraft model.

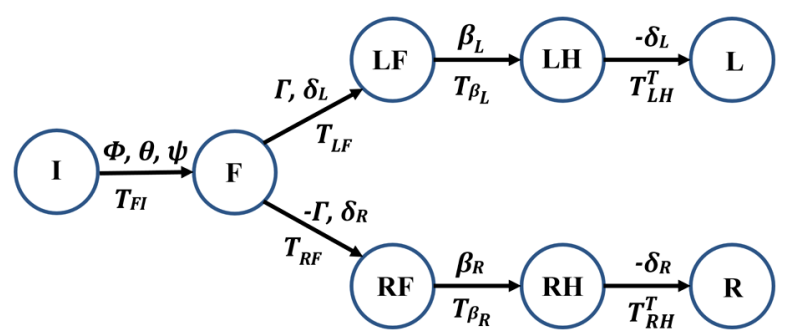

Fig. 2 Frames involved in simulation with rotation angles and transformation matrices between them.
The state vector $\boldsymbol{X}$ of the system is given in Eq. ( $\underline{8})$. Definitions for these state variables follow in Eqs. (9-11). The position, attitude, velocity, and angular-velocity variables are defined for the fuselage body c.g. In addition, the variables $\beta_{L}$ and $\beta_{R}$ are the flap rotations about the joint axis for the left and right wings, respectively.

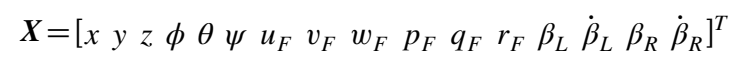

$$
\bar{r}_{O \rightarrow \oplus_{F}}=x \overline{\boldsymbol{I}}_{I}+y \overline{\boldsymbol{J}}_{I}+z \overline{\boldsymbol{K}}_{I}
$$

$$
\begin{gathered}
\bar{V}_{\oplus_{F} / I}=u_{F} \overline{\boldsymbol{I}}_{F}+v_{F} \overline{\boldsymbol{J}}_{F}+w_{F} \overline{\boldsymbol{K}}_{F} \\
\bar{\omega}_{F / I}=p_{F} \overline{\boldsymbol{I}}_{F}+q_{F} \overline{\boldsymbol{J}}_{F}+r_{F} \overline{\boldsymbol{K}}_{F}
\end{gathered}
$$

\section{B. Kinematic Equations of Motion}

The eight-DOF simulation model uses the body-frame components of the fuselage mass-center velocity and angularvelocity vectors rather than inertial-frame measure numbers. The position states are defined in the inertial frame. Kinematic differential equations relate the time derivatives of "position" coordinates to "velocity" coordinates. The kinematic differential equations relating time derivatives of the mass-center position-vector components with the mass-center velocity in the body frame are provided in Eq. (12). In a similar manner, time derivatives of the Euler orientation angles are related to the body-frame angular velocities through Eq. (13).

$$
\begin{gathered}
\left\{\begin{array}{c}
\dot{x} \\
\dot{y} \\
\dot{z}
\end{array}\right\}=\left[T_{F I}\right]^{T}\left\{\begin{array}{c}
u_{F} \\
v_{F} \\
w_{F}
\end{array}\right\} \\
\left\{\begin{array}{c}
\dot{\phi} \\
\dot{\theta} \\
\dot{\psi}
\end{array}\right\}=\left[\begin{array}{ccc}
1 & \sin \phi \tan \theta & \cos \phi \tan \theta \\
0 & \cos \phi & -\sin \phi \\
0 & \sin \phi \sec \theta & \cos \phi \sec \theta
\end{array}\right]\left\{\begin{array}{c}
p_{F} \\
q_{F} \\
r_{F}
\end{array}\right\}
\end{gathered}
$$

\section{Dynamic Equations of Motion}

Using a Newton-Euler approach to form the dynamic equations of motion, the translational and rotational dynamic equations of motion are developed for each of the three bodies (fuselage, left wing, and right wing) separately. The Newton-Euler translational dynamic equations of motion for a general body $B$ are provided in Eq. (14). Likewise, the general body rotational dynamic equations are provided in Eq. (15). For the fuselage body, the vector-derivative transport theorem is used to determine the acceleration and angular momentum in the body frame, Eqs. (15) and (16), respectively. The superscript in front of a derivative term denotes the reference frame. Forces on the fuselage include weight, aerodynamics, propulsive thrust, and joint constraints. Moments on the fuselage about its c.g. include aerodynamics, propulsive torque, elastic stiffness and damping, and joint constraints. 


$$
\begin{aligned}
& m_{B} \bar{a}_{\oplus_{B} / I}=\bar{F} \\
& \frac{{ }^{I} \mathrm{~d} \bar{H}_{B / I}^{\oplus}}{\mathrm{d} t}=\bar{M}_{\oplus} \\
& \bar{a}_{\oplus_{F} / I}=\frac{I \mathrm{~d} \bar{V}_{\oplus_{F} / I}}{\mathrm{~d} t}=\frac{{ }^{F} \mathrm{~d} \bar{V}_{\oplus_{F} / I}}{\mathrm{~d} t}+\bar{\omega}_{F / I} \times \bar{V}_{\oplus_{F} / I} \\
& =\left\{\begin{array}{c}
\dot{u}_{F} \\
\dot{v}_{F} \\
\dot{w}_{F}
\end{array}\right\}+\left\{\begin{array}{c}
p_{F} \\
q_{F} \\
r_{F}
\end{array}\right\} \times\left\{\begin{array}{c}
u_{F} \\
v_{F} \\
w_{F}
\end{array}\right\} \\
& \frac{{ }^{I} \mathrm{~d} \bar{H}_{F / I}^{\oplus}}{\mathrm{d} t}=\frac{{ }^{F} \mathrm{~d} \bar{H}_{F / I}^{\oplus}}{\mathrm{d} t}+\bar{\omega}_{F / I} \times \bar{H}_{F / I}^{\oplus} \\
& =I_{F}\left\{\begin{array}{c}
\dot{p}_{F} \\
\dot{q}_{F} \\
\dot{r}_{F}
\end{array}\right\}+\left\{\begin{array}{c}
p_{F} \\
q_{F} \\
r_{F}
\end{array}\right\} \times I_{F}\left\{\begin{array}{c}
p_{F} \\
q_{F} \\
r_{F}
\end{array}\right\}
\end{aligned}
$$

For the wing equations of motion, all velocity, angular-velocity, acceleration, and angular-acceleration terms are written in terms of the state variables, rather than each body's individual velocity and acceleration terms. To obtain the acceleration of the wing c.g., the kinematic relationship of the accelerations of two points fixed on a rigid body is applied to the wing, Eq. (18), and the fuselage, Eq. (19). Shorthand for position, velocity, and angular-velocity components is introduced in Eqs. (20-24). The components of acceleration of the wing c.g. are written in the wing body frame, so terms in the fuselage body frame are transformed using the matrix relating the fuselage and wing frames, given in Eq. (7). Using that transformation along with the defined shorthand notation and substituting Eq. (19) into Eq. (18), the components of acceleration of the wing are provided in Eq. $(\overline{25})$. The right-wing equations follow analogously to the left-wing equations given.

$$
\bar{a}_{\oplus_{L} / I}=\bar{a}_{\mathrm{LH} / I}+\bar{\omega}_{L / I} \times \bar{\omega}_{L / I} \times \bar{r}_{\mathrm{LH} \rightarrow \oplus_{L}}+\bar{\alpha}_{L / I} \times \bar{r}_{\mathrm{LH} \rightarrow \oplus_{L}}
$$

$$
\bar{a}_{\mathrm{LH} / I}=\bar{a}_{\oplus_{F} / I}+\bar{\omega}_{F / I} \times \bar{\omega}_{F / I} \times \bar{r}_{\oplus_{F} \rightarrow \mathrm{LH}}+\bar{\alpha}_{F / I} \times \bar{r}_{\oplus_{F} \rightarrow \mathrm{LH}}
$$

$$
r_{L}=\mathbb{C}_{F}\left(\bar{r}_{\oplus_{F} \rightarrow \mathrm{LH}}\right)
$$

$$
r_{\mathrm{LL}}=\mathbb{C}_{L}\left(\bar{r}_{\mathrm{LH} \rightarrow \oplus_{L}}\right)
$$

$$
\begin{array}{ll}
V_{F}=\left\{\begin{array}{c}
u_{F} \\
v_{F} \\
w_{F}
\end{array}\right\}, & \omega_{F}=\left\{\begin{array}{c}
p_{F} \\
q_{F} \\
r_{F}
\end{array}\right\}, \\
\dot{V}_{F}=\left\{\begin{array}{c}
\dot{u}_{F} \\
\dot{v}_{F} \\
\dot{w}_{F}
\end{array}\right\}, \quad \dot{\omega}_{F}=\left\{\begin{array}{c}
\dot{p}_{F} \\
\dot{q}_{F} \\
\dot{r}_{F}
\end{array}\right\}
\end{array}
$$

$$
\begin{gathered}
S_{V_{F}}=\mathbb{S}_{F}\left(V_{F}\right)=\left[\begin{array}{ccc}
0 & -w_{F} & v_{F} \\
w_{F} & 0 & -u_{F} \\
-v_{F} & u_{F} & 0
\end{array}\right] \\
S_{\omega_{F}}=\mathbb{S}_{F}\left(\omega_{F}\right), \quad S_{L}=\mathbb{S}_{F}\left(r_{L}\right), \quad S_{\mathrm{LL}}=\mathbb{S}_{L}\left(r_{\mathrm{LL}}\right) \\
a_{L}=T_{\mathrm{LH}}^{T} T_{\beta_{L}} T_{\mathrm{LF}}\left(\dot{V}_{F}+S_{\omega_{F}} V_{F}-S_{L} \dot{\omega}_{F}+S_{\omega_{F}} S_{\omega_{F}} r_{L}\right)-S_{\mathrm{LL}} \dot{\omega}_{L} \\
+S_{\omega_{L}} S_{\omega_{L}} r_{\mathrm{LL}}
\end{gathered}
$$

The preceding equation requires knowledge of the angular velocity and angular acceleration of the wing. Kinematic relationships can be used to define these in terms of the state variables, Eqs. (26-28). Again, all terms are transformed to the wing body frame. These terms are also used in the derivative of angular momentum in the derivation of the rotational dynamic equations of motion for the wing. The derivative of angular momentum of the left wing is provided in Eq. (29), written in component form in Eq. (30).

$$
\bar{\omega}_{L / I}=\bar{\omega}_{L / F}+\bar{\omega}_{F / I}
$$

$$
\begin{gathered}
\omega_{L}=T_{\mathrm{LH}}^{T} T_{\beta_{L}} T_{\mathrm{LF}} \omega_{F}+T_{\mathrm{LH}}^{T} \Lambda \dot{\beta}_{L} \\
\alpha_{L}=\dot{\omega}_{L}=T_{\mathrm{LH}}^{T} T_{\beta_{L}} T_{\mathrm{LF}} \dot{\omega}_{F}+T_{\mathrm{LH}}^{T} \Lambda \ddot{\beta}_{L}+T_{\mathrm{LH}}^{T} \dot{T}_{\beta_{L}} T_{\mathrm{LF}} \omega_{F} \\
\frac{{ }^{\mathrm{d}} \bar{H}_{L / I}^{\oplus}}{\mathrm{d} t}=\frac{{ }^{L} \mathrm{~d} \bar{H}_{L / I}^{\oplus}}{\mathrm{d} t}+\bar{\omega}_{L / I} \times \bar{H}_{L / I}^{\oplus} \\
\mathbb{C}_{L}\left(\frac{{ }^{I} \mathrm{~d} \bar{H}_{L / I}^{\oplus}}{\mathrm{d} t}\right)=I_{L} \dot{\omega}_{L}+S_{\omega_{L}} I_{L} \omega_{L}
\end{gathered}
$$

The wing forces include weight, aerodynamics, and joint constraints. The wing moments about the body c.g. include aerodynamics, elastic stiffness and damping, and joint constraints. The $\Lambda$ and $\Phi$ matrices of Eq. (31) select the appropriate component of the flapping angularvelocity vector and joint constraint moments, respectively.

$$
\Lambda=\left[\begin{array}{l}
1 \\
0 \\
0
\end{array}\right], \quad \Phi=\left[\begin{array}{ll}
0 & 0 \\
1 & 0 \\
0 & 1
\end{array}\right]
$$

The translational and rotational dynamic equations of motion for all three bodies then yield the six equations in component form, Eqs. (32-37).

$$
m_{F}\left(\dot{V}_{F}+S_{\omega_{F}} V_{F}\right)=F_{F_{\text {AERO }}}+F_{\mathrm{THRUST}}+F_{F_{\mathrm{GRAV}}}+F_{\mathrm{LH}}+F_{\mathrm{RH}}
$$

$$
\begin{gathered}
I_{F} \dot{\omega}_{F}+S_{\omega_{F}} I_{F} \omega_{F}=M_{F_{\mathrm{AERO}}}+M_{\mathrm{TORQUE}}-T_{\mathrm{LF}}^{T} \Lambda M_{L_{\mathrm{ELASTIC}}} \\
-T_{\mathrm{LF}}^{T} \Lambda M_{R_{\mathrm{ELASTIC}}}+M_{\mathrm{LH}}+M_{\mathrm{RH}}
\end{gathered}
$$




$$
\begin{gathered}
m_{L} T_{L}\left(\dot{V}_{F}+S_{\omega_{F}} V_{F}-S_{L} \dot{\omega}_{F}+S_{\omega_{F}} S_{\omega_{F}} r_{L}\right)-m_{L} S_{\mathrm{LL}} \dot{\omega}_{L} \\
\quad+m_{L} S_{\omega_{L}} S_{\omega_{L}} r_{\mathrm{LL}}=F_{L_{\mathrm{AERO}}}+F_{L_{\mathrm{GRAV}}}-T_{L} F_{\mathrm{LH}}
\end{gathered}
$$

$$
\begin{aligned}
& I_{L} \dot{\omega}_{L}+S_{\omega_{L}} I_{L} \omega_{L}=M_{L_{\mathrm{AERO}}}+T_{\mathrm{LH}}^{T} \Lambda M_{L_{\mathrm{ELASTIC}}}-T_{L} \Phi_{L} M_{\mathrm{LH}} \\
& \quad-T_{L} S_{L} F_{\mathrm{LH}}
\end{aligned}
$$

$$
\begin{gathered}
m_{R} T_{R}\left(\dot{V}_{F}+S_{\omega_{F}} V_{F}-S_{R} \dot{\omega}_{F}+S_{\omega_{F}} S_{\omega_{F}} r_{R}\right)-m_{R} S_{\mathrm{RR}} \dot{\omega}_{R} \\
\quad+m_{R} S_{\omega_{R}} S_{\omega_{R}} r_{\mathrm{RR}}=F_{R_{\mathrm{AERO}}}+F_{R_{\mathrm{GRAV}}}-T_{R} F_{\mathrm{RH}}
\end{gathered}
$$

$$
\begin{aligned}
& I_{R} \dot{\omega}_{R}+S_{\omega_{R}} I_{R} \omega_{R}=M_{R_{\text {AERO }}}+T_{\mathrm{RH}}^{T} \Lambda M_{R_{\mathrm{ELASTTC}}}-T_{R} \Phi_{R} M_{\mathrm{RH}} \\
& \quad-T_{R} S_{R} F_{\mathrm{RH}}
\end{aligned}
$$

The constraint forces and moments at the joints are of interest to monitor during the simulation, so they are retained in the dynamic equations rather than being algebraically eliminated. This creates a matrix equation consisting of 18 equations and 18 unknowns given in Eqs. (38-45), which is solved for the state derivative vector required for numerical simulation, as well as the joint constraint forces and moments.

$$
[A]\left\{\begin{array}{c}
\dot{V}_{F} \\
\dot{\omega}_{F} \\
\ddot{\beta}_{L} \\
\ddot{\beta}_{R} \\
F_{\mathrm{LH}} \\
F_{\mathrm{RH}} \\
M_{\mathrm{LH}} \\
M_{\mathrm{RH}}
\end{array}\right\}=\left\{\begin{array}{c}
B_{1} \\
B_{2} \\
B_{3} \\
B_{4} \\
B_{5} \\
B_{6}
\end{array}\right\}
$$

in which

$$
A=\left[\begin{array}{ccc}
m_{F} I_{3 \times 3} & 0 & 0 \\
0 & I_{F} & 0 \\
m_{L} T_{L} & -m_{L} T_{L} S_{L}-m_{L} S_{\mathrm{LL}} T_{L} & -m_{L} S_{\mathrm{LL}} T_{\mathrm{LH}}^{T} \Lambda_{L} \\
0 & I_{L} T_{L} & I_{L} T_{\mathrm{LH}}^{T} \Lambda_{L} \\
m_{R} T_{R} & -m_{R} T_{R} S_{R}-m_{R} S_{\mathrm{RR}} T_{R} & 0 \\
0 & I_{R} T_{R} & 0
\end{array}\right.
$$

$$
B_{1}=-m_{F} S_{\omega_{F}} V_{F}+F_{F}
$$

$$
B_{2}=-S_{\omega_{F}} I_{F} \omega_{F}+M_{F}
$$

$$
\begin{aligned}
B_{3} & =-m_{L} T_{L}\left(S_{\omega_{F}} V_{F}+S_{\omega_{F}} S_{\omega_{F}} r_{L}\right)+m_{L} S_{\mathrm{LL}} T_{\mathrm{LH}}^{T} \dot{T}_{\beta_{L}} T_{\mathrm{LF}} \omega_{F} \\
& -m_{L} S_{\omega_{L}} S_{\omega_{L}} r_{\mathrm{LL}}+F_{L}
\end{aligned}
$$

$$
B_{4}=-I_{L} T_{\mathrm{LH}}^{T} \dot{T}_{\beta_{L}} T_{\mathrm{LF}} \omega_{F}-S_{\omega_{L}} I_{L} \omega_{L}+M_{L}
$$

$$
\begin{aligned}
B_{5} & =-m_{R} T_{R}\left(S_{\omega_{F}} V_{F}+S_{\omega_{F}} S_{\omega_{F}} r_{R}\right)+m_{R} S_{\mathrm{RR}} T_{\mathrm{RH}}^{T} \dot{T}_{\beta_{R}} T_{R F} \omega_{F} \\
& -m_{R} S_{\omega_{R}} S_{\omega_{R}} r_{\mathrm{RR}}+F_{R}
\end{aligned}
$$

$$
B_{6}=-I_{R} T_{\mathrm{RH}}^{T} \dot{T}_{\beta_{R}} T_{\mathrm{RF}} \omega_{F}-S_{\omega_{R}} I_{R} \omega_{R}+M_{R}
$$

The $[A]$ matrix in Eqs. (38) and (39) is a square $6 \times 6$ block matrix, in which each block consists of a $3 \times 3$ matrix. The $B$ matrix is a $6 \times 1$ block vector, in which each element consists of three rows.

\section{Applied Loads}

In the preceding dynamic equations of motion, the total externally applied forces and moments about the mass center in the individual body reference frame appear, such as $F_{F}$ and $M_{F}$ for the fuselage body, and $F_{L}$ and $M_{L}$ for the left wing. These terms do not include the connection constraint loads, but are comprised of the forces and moments due to gravity, aerodynamics, propulsion, and joint elasticity. The propulsive force and moment consist of the thrust and torque generated by the propulsive system.

\section{Elastic Loads}

The compliant hinge is modeled as a linear rotational spring and damper, generating a moment about the hinge axis when the flap angle of the wing is deflected from the zero-load rotation angle of the spring $\beta_{0}$. This moment is applied equally and opposite between the fuselage and wing bodies. The component of the elastic moment in the joint frame is given in Eq. (46), in which $k$ is the spring stiffness and $c$ is the damping coefficient.

$$
M_{L_{\mathrm{ELASTIC}}}=-k\left(\beta_{L}-\beta_{0}\right)-c \dot{\beta}_{L}
$$

\section{Aerodynamic Loads}

Aerodynamic loads on the aircraft are computed using superposition. In the preceding equations of motion, the aerodynamic forces and moments are determined for each body individually. For the fuselage body, which consists of the fuselage, vertical tail, and horizontal tail, a stability-derivative formulation is employed. For the left and right wings, a nonlinear lifting-line approach is used. Both models are described as follows.

Analytical stability derivatives are used for the fuselage, as well as analytical estimates for the conventional control derivatives. The lift and drag forces, Eqs. (47) and (48), respectively, are determined by the lift and drag on the fuselage, vertical tail, and horizontal tail. In Eq. (48), $e$ is Oswald's efficiency factor, and $\mathscr{R}$ is the tail aspect ratio. The first three terms of the drag model, Eq. (48), represent the twodimensional (2-D) airfoil drag from the tail lifting surface. The fuselage drag is also included in the first term, $C_{D 0}$. The fourth term, which is dependent on the square of the lift coefficient, adds the threedimensional finite wing, or induced drag, effects. The side-force coefficient is given in Eq. (49). The lift and drag forces are rotated through the angle of attack to transform them to the body frame, Eq. (50). The coefficients of roll, pitch, and yaw moments are presented in Eqs. (51-53). The body-frame forces and moments are 
calculated from the coefficients using Eq. (54), in which $S$ is the wing area, $b$ is the wingspan, and $\bar{c}$ is the mean aerodynamic chord.

$$
C_{L}=C_{L_{0}}+C_{L_{\alpha}} \alpha+C_{L_{\delta_{\mathrm{LE}}}} \delta_{\mathrm{LE}}+C_{L_{\delta_{\mathrm{RE}}}} \delta_{\mathrm{RE}}
$$

$C_{D}=C_{D_{0}}+C_{D_{\alpha}} \alpha+C_{D_{\alpha^{2}}} \alpha^{2}+\frac{C_{L}^{2}}{\pi e A R}+C_{D_{\delta_{L E}}} \delta_{L E}+C_{D_{\delta_{R E}}} \delta_{R E}$

$$
C_{Y}=C_{Y_{\beta}} \beta+C_{Y_{p}} \hat{p}+C_{Y_{r}} \hat{r}+C_{Y_{\delta_{R}}} \delta_{R}
$$

$$
\begin{aligned}
& \left\{\begin{array}{l}
C_{X} \\
C_{Y} \\
C_{Z}
\end{array}\right\}=\left[\begin{array}{ccc}
\cos (\alpha) & 0 & -\sin (\alpha) \\
0 & 1 & 0 \\
\sin (\alpha) & 0 & \cos (\alpha)
\end{array}\right]\left\{\begin{array}{c}
-C_{D} \\
C_{Y} \\
-C_{L}
\end{array}\right\} \\
& C_{l}=C_{l_{\beta}} \beta+C_{l_{p}} \hat{p}+C_{l_{r}} \hat{r}+C_{l_{\delta_{\mathrm{LE}}}} \delta_{\mathrm{LE}}+C_{l_{\delta_{\mathrm{RE}}}} \delta_{\mathrm{RE}}+C_{l_{\delta_{R}}} \delta_{R} \\
& C_{m}=C_{m_{0}}+C_{m_{\alpha}} \alpha+C_{m_{q}} \hat{q}+C_{m_{\delta_{\mathrm{LE}}}} \delta_{\mathrm{LE}}+C_{m_{\delta_{\mathrm{LE}}}} \delta_{\mathrm{LE}} \\
& C_{n}=C_{n_{\beta}} \beta+C_{n_{p}} \hat{p}+C_{n_{r}} \hat{r}+C_{n_{\delta_{\mathrm{LE}}}} \delta_{\mathrm{LE}}+C_{n_{\delta_{\mathrm{RE}}}} \delta_{\mathrm{RE}}+C_{n_{\delta_{R}}} \delta_{R} \\
& \left\{\begin{array}{c}
X \\
Y \\
Z \\
L \\
M \\
N
\end{array}\right\}=\frac{1}{2} \rho_{\infty} V_{\infty}^{2} S\left\{\begin{array}{c}
C_{X} \\
C_{Y} \\
C_{Z} \\
b C_{l} \\
\bar{c} C_{m} \\
b C_{n}
\end{array}\right\}
\end{aligned}
$$

The aerodynamic loads on each wing are calculated using a numerical lifting-line method, as detailed in [35]. The wing is divided into spanwise stations, forming panels, as depicted in Fig. 3. A node point is placed at the quarter chord of each station. Each panel has a horseshoe vortex with circulation strength of $\Gamma_{p}$. The bound portion of the horseshoe vortex is placed along the quarter chord connecting the node points. The trailing vortices of the horseshoe vortex are aligned with the freestream velocity direction. A control point is placed on each panel at the quarter chord midway between nodes. The downwash at an arbitrary control point from any horseshoe vortex is obtained using the Biot-Savart law. The vectors $\overline{\boldsymbol{r}}_{1}$ and $\overline{\boldsymbol{r}}_{2}$ are defined

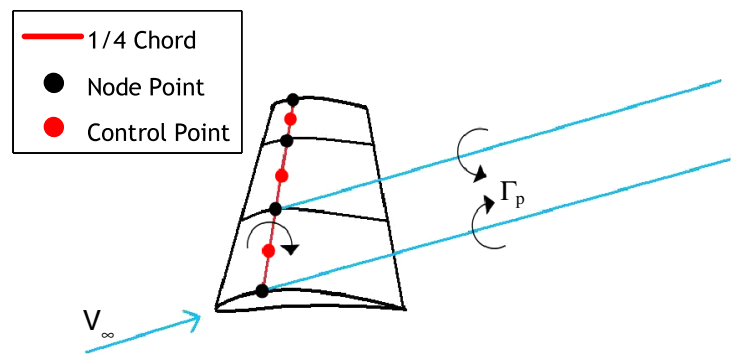

Fig. 3 Spanwise panels and example horseshoe vortex for numerical lifting-line method. from each node point of a horseshoe vortex to the control point. Notation for the magnitudes of these vectors is provided in Eq. (55). The total downwash at the control point from the horseshoe vortex can be written by summing the downwash contribution from each vortex filament of the horseshoe, Eq. (56). It is of note that a bound vortex does not produce downwash along its length, and the second term in the brackets of this equation, which is the downwash contribution from the bound vortex, will produce a singularity when $\overline{\boldsymbol{r}}_{1}$ and $\overline{\boldsymbol{r}}_{2}$ are collinear and opposite directions. This is the case in Fig. 4, when the control point investigated is on the same panel as the horseshoe vortex. In such cases, Eq. (57) must be used to avoid the singularity. The effective angle of attack that determines the lift on the local airfoil section is found from Eq. (58). It is important to note that the freestream velocity components in this equation include aerodynamic velocity imparted by the motion of the body and atmospheric wind, if any exists. The induced velocity components in Eq. (58) are described in the local-airfoil-section body frame. However, Eqs. (56) and (57) are easiest to compute with the vectors expressed in the inertial frame. Equation (59) relates the inertialframe downwash-vector components to the local-frame downwash components.

$$
r_{1}=\left\|\overline{\boldsymbol{r}}_{1}\right\|, \quad r_{2}=\left\|\overline{\boldsymbol{r}}_{2}\right\|
$$

$$
\bar{\varepsilon}=\frac{\Gamma_{p}}{4 \pi}\left[\frac{\overline{\boldsymbol{V}}_{\infty} \times \overline{\boldsymbol{r}}_{2}}{r_{2}\left(r_{2}-\overline{\boldsymbol{V}}_{\infty} \cdot \overline{\boldsymbol{r}}_{2}\right)}+\frac{\left(r_{1}+r_{2}\right)\left(\overline{\boldsymbol{r}}_{1} \times \overline{\boldsymbol{r}}_{2}\right)}{r_{1} r_{2}\left(r_{1} r_{2}+\overline{\boldsymbol{r}}_{1} \cdot \overline{\boldsymbol{r}}_{2}\right)}-\frac{\overline{\boldsymbol{V}}_{\infty} \times \overline{\boldsymbol{r}}_{1}}{r_{1}\left(r_{1}-\overline{\boldsymbol{V}}_{\infty} \cdot \overline{\boldsymbol{r}}_{1}\right)}\right]
$$

$$
\overline{\boldsymbol{\varepsilon}}=\frac{\Gamma_{p}}{4 \pi}\left[\frac{\overline{\boldsymbol{V}}_{\infty} \times \overline{\boldsymbol{r}}_{2}}{r_{2}\left(r_{2}-\overline{\boldsymbol{V}}_{\infty} \cdot \overline{\boldsymbol{r}}_{2}\right)}-\frac{\overline{\boldsymbol{V}}_{\infty} \times \overline{\boldsymbol{r}}_{1}}{r_{1}\left(r_{1}-\overline{\boldsymbol{V}}_{\infty} \cdot \overline{\boldsymbol{r}}_{1}\right)}\right]
$$

$$
\alpha_{e}=\tan ^{-1}\left(\frac{w_{\infty}-w_{i}}{u_{\infty}+u_{i}}\right)
$$

$$
\left\{\begin{array}{c}
u_{i} \\
v_{i} \\
w_{i}
\end{array}\right\}=T_{I B}^{T} \mathbb{C}_{I}(\overline{\boldsymbol{\varepsilon}})
$$

The calculation to include the wake into the contribution to aerodynamic loads from lifting surfaces does not have a closedform solution for arbitrary configurations. An iterative, numerical approach is adopted [36,37]. An initial spanwise downwash distribution is assumed at all control points. Using this initial downwash, the lift, drag, and pitching moment are calculated at each panel using 2-D airfoil data. From the panel lift, the circulation strength of the horseshoe vortex at each panel is calculated using Eq. (60), in which $L_{p}^{\prime}$ is the sectional lift, $c_{l}$ is the sectional lift coefficient, and $\bar{c}_{p}$ is the mean aerodynamic chord of the panel. Using

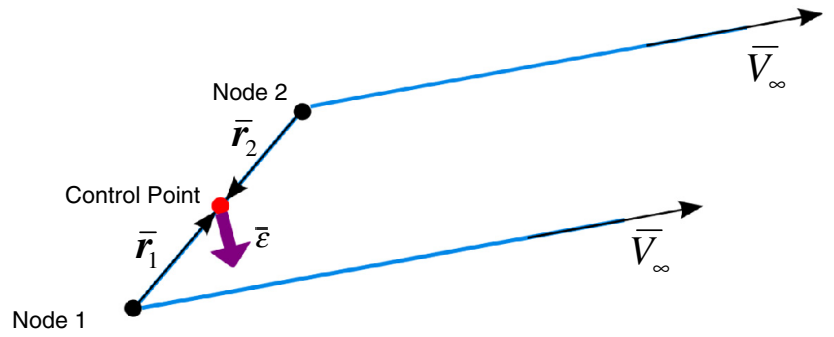

Fig. 4 Influence of horseshoe vortex on control point. 
Eqs. (56) and (57), the downwash contribution from every horseshoe vortex on each control point is calculated and summed to compute a new downwash distribution. The new distribution is compared to the previous, and if the difference is within the specified convergence criteria, the solution is considered to be converged. Otherwise, the newly calculated distribution is damped, as in Eq. (61), and used as the initial distribution, and the process iterates until convergence is achieved [36,37]. Once convergence is reached, the induced angle of attack is calculated at each station, which is then used to obtain the effective angle of attack. With knowledge of the 2-D airfoil, the section lift, drag, and pitching moment of each panel are obtained. The contribution of each panel to the aerodynamic forces and moments about the wing c.g. in the body frame is then summed over all the panels on the wing.

$$
\begin{gathered}
\Gamma_{p}=\frac{L_{p}^{\prime}}{\rho_{\infty} V_{\infty}}=\frac{1}{2} V_{\infty} \bar{c}_{p} c_{l} \\
\overline{\boldsymbol{\varepsilon}}_{\text {input }}=\overline{\boldsymbol{\varepsilon}}_{\text {old }}+\zeta\left(\overline{\boldsymbol{\varepsilon}}_{\text {new }}-\overline{\boldsymbol{\varepsilon}}_{\text {old }}\right)
\end{gathered}
$$

\section{E. Flying-Quality Analysis}

Linear dynamic models of the aircraft system are generated from the nonlinear equations of motion about a steady, level-flight condition using small perturbation theory [38]. The linear models are used to perform modal analysis. The ability of an aircraft to perform various categories of missions and the phases of flight composing the mission without undue stress on the operator are measured by the flying-qualities criteria defined in MIL-F-8785C [39]. These criteria are determined from the natural frequency and damping of the lowfrequency flight dynamic modes of the vehicle.

In the military specifications, the short-period-mode flying qualities are also quantified by the parameters acceleration sensitivity and control anticipation parameter (CAP). The acceleration sensitivity $\eta_{\alpha}$ is the normal load factor per unit angle of attack of the vehicle, which is generally a fixed quantity for an aircraft not related to the dynamic response, since it only depends on the weight and lift-curve slope of the vehicle. The CAP uses the natural frequency of the short-period mode in its formulation.

$$
\begin{gathered}
\eta_{\alpha}=\frac{C_{L_{\alpha}}}{C_{W}} \\
\mathrm{CAP}=\frac{\omega_{n_{\mathrm{SP}}}^{2}}{\eta_{\alpha}}
\end{gathered}
$$

To elucidate how compliant hinges change the flight dynamic modes of the vehicle, the changes in the aerodynamic stability derivatives are investigated using static aeroelasticity analysis, such as that described in [40]. Assuming quasi-static wing flapping, the flap rates, $\dot{\beta}_{L}$ and $\dot{\beta}_{R}$, and acceleration are zero, and the flap angles $\beta_{L}$ and $\beta_{R}$ can be determined from the linear model in terms of the other state variables. These can then be substituted into the numerically calculated aerodynamic stability derivatives to determine the effect of flexibility on the stability derivatives that significantly contribute to the traditional dynamic modes of the vehicle. For the short-period mode, the modal natural frequency is largely determined by the stability derivatives $M_{w}, Z_{w}$, and $M_{q}$. These terms are all negative and will increase the natural frequency of the mode as their magnitudes increase. The stability derivatives $Z_{w}$ and $M_{q}$ contribute to the damping of the short-period mode, increasing the damping as they become more negative. The natural frequency of the phugoid is most affected by $Z_{u}$, which increases the natural frequency as it becomes more negative. The damping of the mode is affected by $X_{u}$, with damping increased by more negative values of $X_{u}$, as well as the natural frequency of the mode. In the longitudinal modes, increases in the natural frequency of a mode will reduce the mode damping. The roll mode, which is nonoscillatory, is determined by $L_{p}$ and $N_{p}$. The roll-damping term $L_{p}$ stabilizes the mode and reduces the time to half as it becomes more negative. The term $N_{p}$ is destabilizing to the mode if it is positive, increasing the time to half of the mode, and stabilizing if it is negative. Likewise, the contributions to the spiral mode include $N_{r}, L_{r}, N_{v}$, and $L_{v}$ with the weathercock stability $N_{v}$, destabilizing the mode, and the dihedral effect $L_{v}$, stabilizing the mode. The natural frequency of the Dutch-roll mode increases with larger weathercock stability and dihedral. Yaw damping, $N_{r}$, and weathercock stability derivatives increase damping and stabilize the mode, whereas increasing dihedral decreases damping to destabilize the mode.

\section{Results}

To explore the ability of discrete structural hinges to tailor flight dynamic behavior, results are generated for an example aircraft.

\section{A. Aircraft}

The example is a small, conventional unmanned aircraft. It is pictured, along with reference frames assigned to each body, in Fig. 1. The mass of the aircraft is $0.84 \mathrm{~kg}$. The wingspan is $0.8 \mathrm{~m}$, and the mean aerodynamic chord is $0.082 \mathrm{~m}$. The aircraft has a cruise speed of $17.3 \mathrm{~m} / \mathrm{s}$. The acceleration sensitivity of the aircraft is $7.3 \mathrm{rad}^{-1}$.

The wings of the aircraft are hinged, permitting rotation about the joint axis. Elasticity of the discrete structural joint is modeled with rotational springs and dampers. The nominal aircraft configuration has the wings' elastic hinges at the wing root and attached to the top center of the fuselage. The elastic joint spring stiffness is $10 \mathrm{~N} \cdot \mathrm{m} / \mathrm{rad}$, and joint damping coefficient is $0.30 \mathrm{~N} \cdot \mathrm{m} /(\mathrm{rad} / \mathrm{s})$. Parameters are varied about this nominal configuration. The spring stiffness is varied from 0.5 to $75 \mathrm{~N} \cdot \mathrm{m}$ in increments of $1 \mathrm{~N} \cdot \mathrm{m}$, and the damping coefficient is varied from 0.05 to $1.0 \mathrm{~N} \cdot \mathrm{m} /(\mathrm{rad} / \mathrm{s})$ in increments of $0.05 \mathrm{~N} \cdot \mathrm{m} /(\mathrm{rad} / \mathrm{s})$.

\section{B. Compliant-Hinge Configurations}

In the nominal configuration, the hinges are aligned with the $\boldsymbol{I}$ axes of each wing and the fuselage body. In one configuration study, the vertical location of the hinge where the roots of the wings attach to the fuselage is varied, whereas the horizontal location of the hinge remains in the center of the fuselage. Keeping with the traditional aircraft body-fixed frame, a positive distance is below the fuselage c.g. The attachment point is varied from $-0.030 \mathrm{~m}$ (top of the fuselage) to $0.030 \mathrm{~m}$ (bottom of the fuselage) by increments of $0.002 \mathrm{~m}$. Figure $5 \mathrm{a}$ is a diagram depicting the variation in the vertical hinge location on the fuselage.

The hinges can also be oriented so that the pitch or sweep of the wing will couple with the dihedral change, known as pitch-flap coupling or sweep-flap coupling. This is achieved by rotating the hinge axis by the angle $\delta_{3}$, as shown in Fig. $\underline{5 b}$ for pitch-flap coupling, or by the angle $\delta_{2}$, Fig. 5c, for sweep-flap coupling. In the case of pitch-flap coupling, the angle of attack will increase or decrease as the wing is deflected. For both $\delta_{3}$ and $\delta_{2}$ hinge orientations, the hinge-axis angles are varied from -90 to $+90 \mathrm{deg}$ by increments of $1 \mathrm{deg}$. The effect of moving the location of the hinge along the span of the wing, thereby changing the area of the wing section being articulated, is also studied. This hinge offset is shown as the distance $e$ in Fig. 5b. Each wing is divided into eight uniform sections, and the hinge is placed at each of these division points. Thus, for a half-span of $0.40 \mathrm{~m}$, the hinge is varied from 0 to $0.35 \mathrm{~m}$ in increments of $0.05 \mathrm{~m}$.

\section{Trim Controller}

The rigid version of the aircraft has four controls: throttle, rudder, right elevon, and left elevon. These are used to achieve basic trim of the vehicle. The elevon commands are determined by mixing the elevator and aileron commands computed by a trim controller. To achieve a straight-and-level trim condition, a simple four-channel proportional-integral (PI) trim controller is used. Throttle is used to 


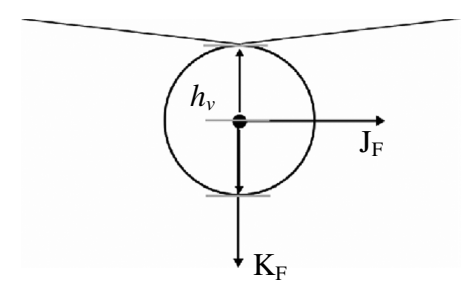

a)

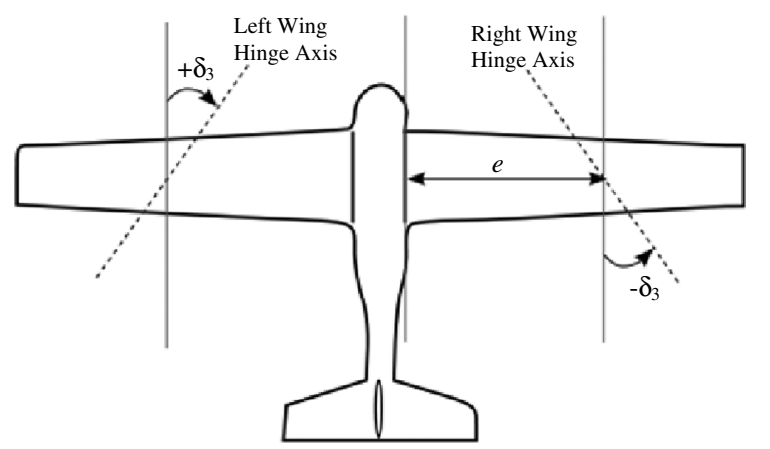

b)

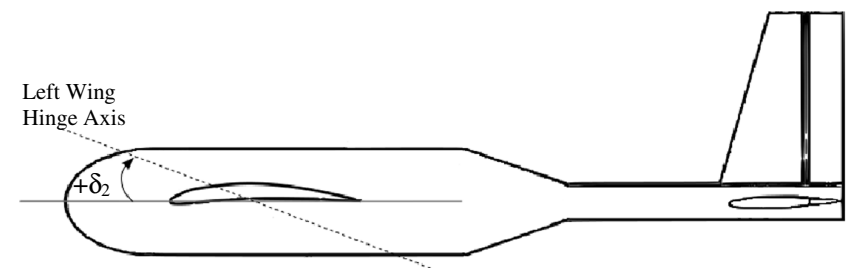

c)

Fig. 5 Configuration parameters: a) vertical hinge location, b) spanwise hinge location and $\delta_{3}$ hinge orientation, and c) $\delta_{2}$ hinge orientation.

control velocity to a commanded value. Ailerons are used to zero the bank angle $\phi$, and rudder is used to zero sideslip $v$. Elevators are used to hold a commanded altitude. To calculate the elevator command, a PI controller based on the altitude error is first used to generate a pitch-angle command. The pitch-angle command is then fed into a proportional controller to determine the elevator angle. In addition to the trim controller, another controller changes the zero-load angle of the joint rotational spring, so that the wings have $3 \mathrm{deg}$ dihedral in trim for equal comparison of all cases, since lower spring-stiffness values naturally allow the wings to increase dihedral. This ensures that the linear models are generated about the same vehicle trim state regardless of hinge design parameters.

\section{Stability Analysis}

For each parameter variation, a linear model is generated about a steady, level trim condition. The dynamics matrix, or state matrix, of the linear dynamic system consists of partial derivatives of the state equations evaluated at the trim state. These partial derivatives are obtained numerically using fourth-order central differencing. For numerical-derivative calculations, the position states are perturbed by

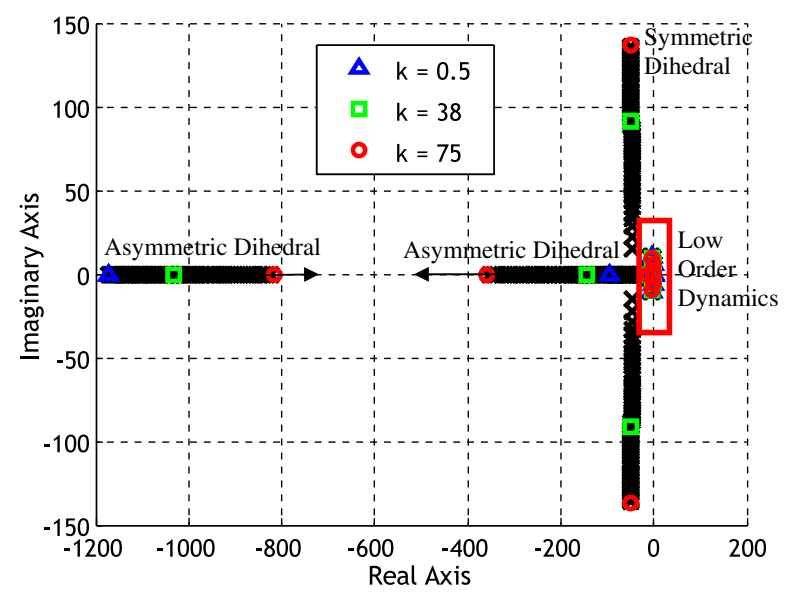

Fig. 6 Root locus as a function of discrete structural-hinge spring stiffness.

a value of $1.0 \times 10^{-4}$, attitude states by $10^{-5}$, and velocity and angular-velocity rates by $10^{-6}$. Performing eigenanalysis on the linear-model state matrix, root-locus plots are generated to depict how the modes change as the discrete structural-hinge parameters are varied. Table 1 summarizes the effect of changes in each hinge design parameter on the flight dynamic modes of the vehicle. Ranges of natural frequency, damping, or time to half (double) are given for each mode, as appropriate. A dash in the table represents when the hinge parameter does not affect the mode (variations less than 1\%). Overall, the longitudinal modes are more affected by variations in properties of the discrete structural hinge than the lateral modes. The $\delta_{3}$ orientation has the largest impact on the modal properties, whereas hinge damping has the smallest effect. More details on each trade study, analysis of the root loci, and the effect on handling qualities are provided as follows.

\section{Effect of Spring Stiffness}

The spring stiffness $k$ is varied from 0.5 to $75 \mathrm{~N} \cdot \mathrm{m} / \mathrm{rad}$, first from 0.5 to $1 \mathrm{~N} \cdot \mathrm{m} / \mathrm{rad}$, and then in increments of $1 \mathrm{~N} \cdot \mathrm{m} / \mathrm{rad}$. As the spring stiffness is increased, the aircraft becomes more similar to a rigid aircraft. Statically (on the ground, no wind), at the softest spring stiffness of $0.5 \mathrm{~N} \cdot \mathrm{m} / \mathrm{rad}$, the wings droop $19.2 \mathrm{deg}$ from their own weight, whereas for the stiffest spring value of $75 \mathrm{~N} \cdot \mathrm{m} / \mathrm{rad}$, wing droop is $0.14 \mathrm{deg}$. At the nominal, moderate spring stiffness of $10 \mathrm{~N} \cdot \mathrm{m} / \mathrm{rad}$, wing droop is $1 \mathrm{deg}$. In the root locus of Fig. 6 , there are two distinct very-high-order modes. These are the two modes added to the system by the two additional DOF of the articulated wings. The asymmetric dihedral mode is an overdamped mode consisting of two real eigenvalues. At high, almost rigid, stiffness values, the symmetric dihedral mode is a very high-frequency oscillatory mode, but as $k$ is decreased, the eigenvalues move toward each other to an eventual break-in point, after which the mode is overdamped with two real eigenvalues. The symmetric flap mode breaks in into a real mode between spring stiffnesses of 8 and $9 \mathrm{~N} \cdot \mathrm{m} / \mathrm{rad}$.

Focusing on the low-frequency dynamics in Fig. 7, spring stiffness of the discrete structural hinge affects the traditional dynamic modes

Table 1 Summary of mode changes by varying parameters

\begin{tabular}{|c|c|c|c|c|c|c|c|c|}
\hline & \multicolumn{2}{|c|}{ Short period } & \multicolumn{2}{|c|}{ Phugoid } & \multicolumn{2}{|c|}{ Dutch roll } & \multirow{2}{*}{$\frac{\text { Roll }}{t_{1 / 2}}$} & \multirow{2}{*}{$\begin{array}{l}\text { Spiral } \\
t_{\text {double }} \\
\end{array}$} \\
\hline & $\omega_{n}$ & $\zeta$ & $\omega_{n}$ & $\zeta$ & $\omega_{n}$ & $\zeta$ & & \\
\hline Spring stiffness & $10.7-12$ & $0.415-0.499$ & $0.603-0.614$ & $0.11-0.21$ & -- & -- & $0.0516-0.0531$ & -- \\
\hline Hinge damping & $10.6-11$ & $0.44-0.46$ & -- & -- & -- & -- & $0.052-0.0537$ & -- \\
\hline Vertical hinge location & $7.05-10.72$ & $0.462-0.738$ & $0.604-0.718$ & $0.154-0.211$ & - & $0.087-0.091$ & $0.0517-0.0526$ & -- \\
\hline Spanwise hinge location & -- & $0.421-0.462$ & -- & $0.211-0.215$ & -- & -- & -- & -- \\
\hline$\delta_{3}$ Orientation & $8.69-11.9$ & $0.32-1.01$ & $0.435-0.959$ & $0.064-0.30$ & -- & $0.0897-0.0914$ & $0.050-0.053$ & $4.64-4.84$ \\
\hline$\delta_{2}$ Orientation & $9.09-11.7$ & $0.346-0.651$ & $0.504-0.757$ & $0.151-0.259$ & -- & $0.0912-0.0927$ & -- & -- \\
\hline
\end{tabular}


of the aircraft. The most notable changes occur in the short-period mode. Table 1 provides ranges for changes in the short-period mode, but examining the root locus plots in Fig. 8, it is apparent that it is not possible to achieve any combination of damping and natural frequency. As stiffness is decreased, the short-period mode first increases damping while decreasing natural frequency. Then, the

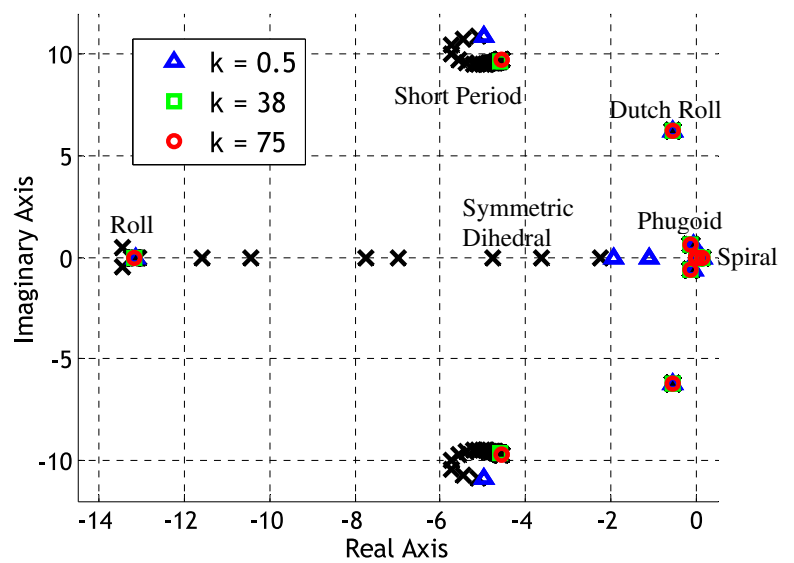

Fig. 7 Root locus of traditional flight dynamic modes as a function of spring stiffness.
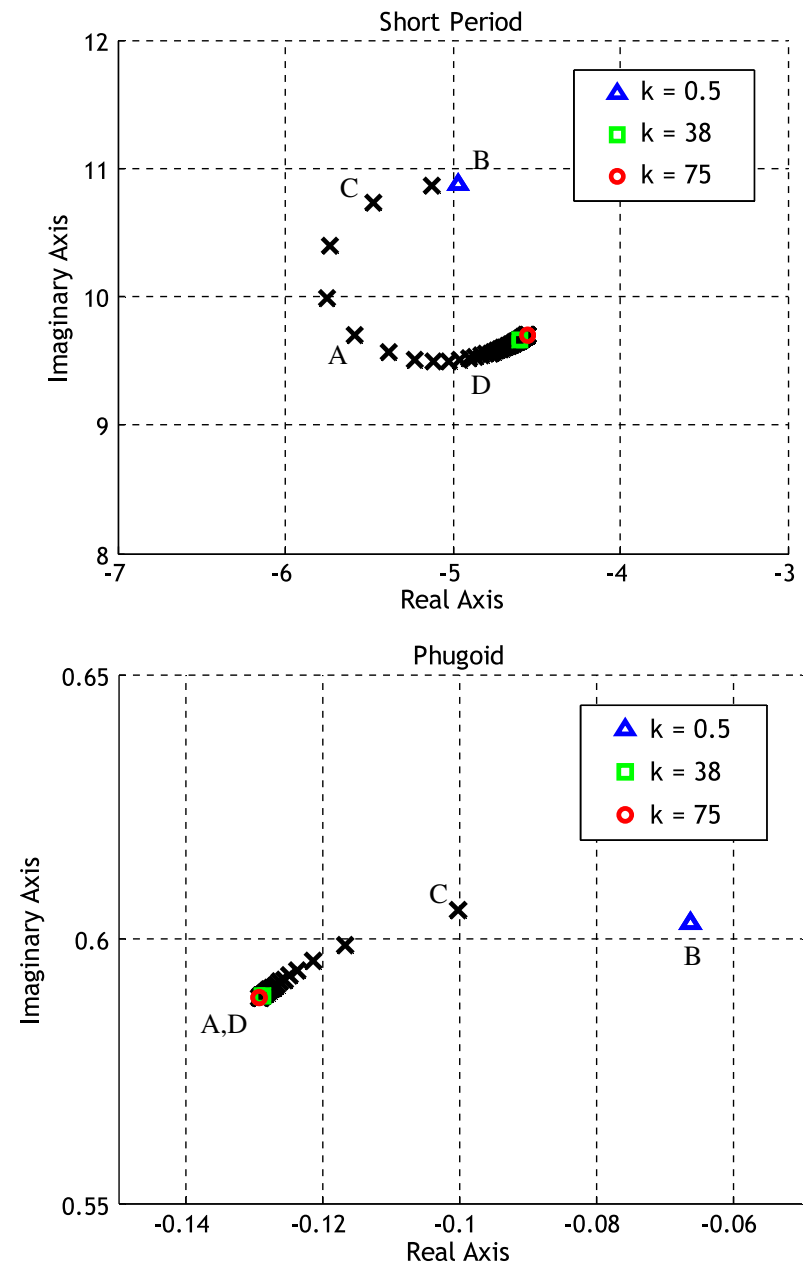

Fig. 8 Spring stiffness variation; A: maximum damping, B: minimum damping, C: maximum natural frequency, D: minimum natural frequency. natural frequency increases while damping is decreased. The eigenvalues representing the maximum and minimum damping and natural frequency are noted on the plots with letters A, B, C, and D. For the phugoid, mode damping is impacted much more than the natural frequency. At the highest stiffness considered, the mode is at its maximum damping and minimum natural frequency, and as the stiffness is decreased, the damping decreases and natural frequency increases. The roll mode is not significantly impacted by the change in stiffness. Interestingly, though, at a stiffness of $4 \mathrm{~N} \cdot \mathrm{m} / \mathrm{rad}$, the roll mode mixes with one of the asymmetric flap modes to create a new slightly oscillatory flap-rolling mode, seen in Fig. 7. The mode is extremely highly damped, however, so it does not alter the flight dynamic response to control input significantly.

These changes in modal frequency and damping correspond to changes in the flying qualities of the aircraft. In the short-period mode, changing the stiffness through a wide range yields a $12.1 \%$ change in the undamped natural frequency. This produces a $25.8 \%$ change in the CAP. The damping ratio of the short-period mode changes by $20.2 \%$. Handling qualities for the phugoid are given as a minimum damping ratio. The range of damping of the phugoid varies by $90.9 \%$ from the lowest damping ratio at a spring stiffness of $0.5 \mathrm{~N} \cdot \mathrm{m} / \mathrm{rad}$ to the highest damping ratio at the highest spring stiffness of $75 \mathrm{~N} \cdot \mathrm{m} / \mathrm{rad}$. The lowest damping ratio is still well above the minimum for Level 1 performance in this case, so the phugoid handling qualities can be traded off for better performance in the short-period mode when using compliant hinges. The only lateral mode affected by the spring stiffness is the roll mode, for which handling-quality criteria are determined by the maximum time constant of the mode. The roll-mode time constant varies $3.75 \%$, mostly due to the mixing of the flap mode with the roll mode. The spiral and Dutch-roll modes are not affected by variations in spring stiffness.

From static aeroelastic analysis of the stability derivatives, the increase in natural frequency of the short-period mode due to decreasing stiffness is largely driven by the pitch stiffness, $M_{w}$, becoming more negative. The decrease in damping of the mode with decreasing stiffness is a result of the increase in natural frequency, as well as the $Z_{w}$ stability derivative becoming less negative. These stability derivatives vary nonlinearly with spring stiffness. With an increase in vertical velocity $w$, flexible hinges allow the wings to flap up, increasing the dihedral. This reduces the $Z$ aerodynamic force of the wing, thereby also reducing the pitch-moment contribution from the wing because the c.g. of the vehicle is located aft of the main wing aerodynamic center. The phugoid mode is largely affected by the stability derivatives $X_{u}$ and $Z_{u}$. As spring stiffness is decreased, $X_{u}$ becomes more negative by $5 \%$ and $Z_{u}$ becomes less negative by $7 \%$. These changes suggest an increase in the damping of the phugoid mode, but the opposite trend is seen, whereby the damping of the phugoid mode is significantly reduced with hinge flexibility. This suggests that coupling of the acceleration and velocity terms, such as the flapping rate of the wings, which is not accounted for in the static aeroelastic analysis, plays the largest role in the phugoid response.

\section{Effect of Damping Coefficient}

The damping constant is varied from 0.05 to $1.0 \mathrm{~N} \cdot \mathrm{m} /(\mathrm{rad} / \mathrm{s})$ by $0.05 \mathrm{~N} \cdot \mathrm{m} /(\mathrm{rad} / \mathrm{s})$. Of the traditional low-frequency flight dynamic modes, the short-period and roll modes are most affected by the damping coefficient, Fig. 9. The points corresponding to the maximum and minimum mode damping and natural frequency of the short period are labeled on the graph. The roll mode again mixes with the low-order overdamped asymmetric flap mode to create a new slightly oscillatory flap-rolling mode. This occurs at the same spring stiffness to damping ratio in this case as in the variable spring-stiffness case. To better show the modal behavior, a finer increment is used between the damping ratios of 0.7 and $0.8 \mathrm{~N} \cdot \mathrm{m} /(\mathrm{rad} / \mathrm{s})$. While the new mode remains nearly critically damped, there is a noticeable change in the time to half of the mode.

In relation to handling qualities, the natural frequency of the shortperiod mode increases by $3.77 \%$ across the range of damping ratios tested, corresponding to a $7.69 \%$ increase in the CAP. The damping ratio of the short-period mode can be increased by $4.54 \%$. The only 

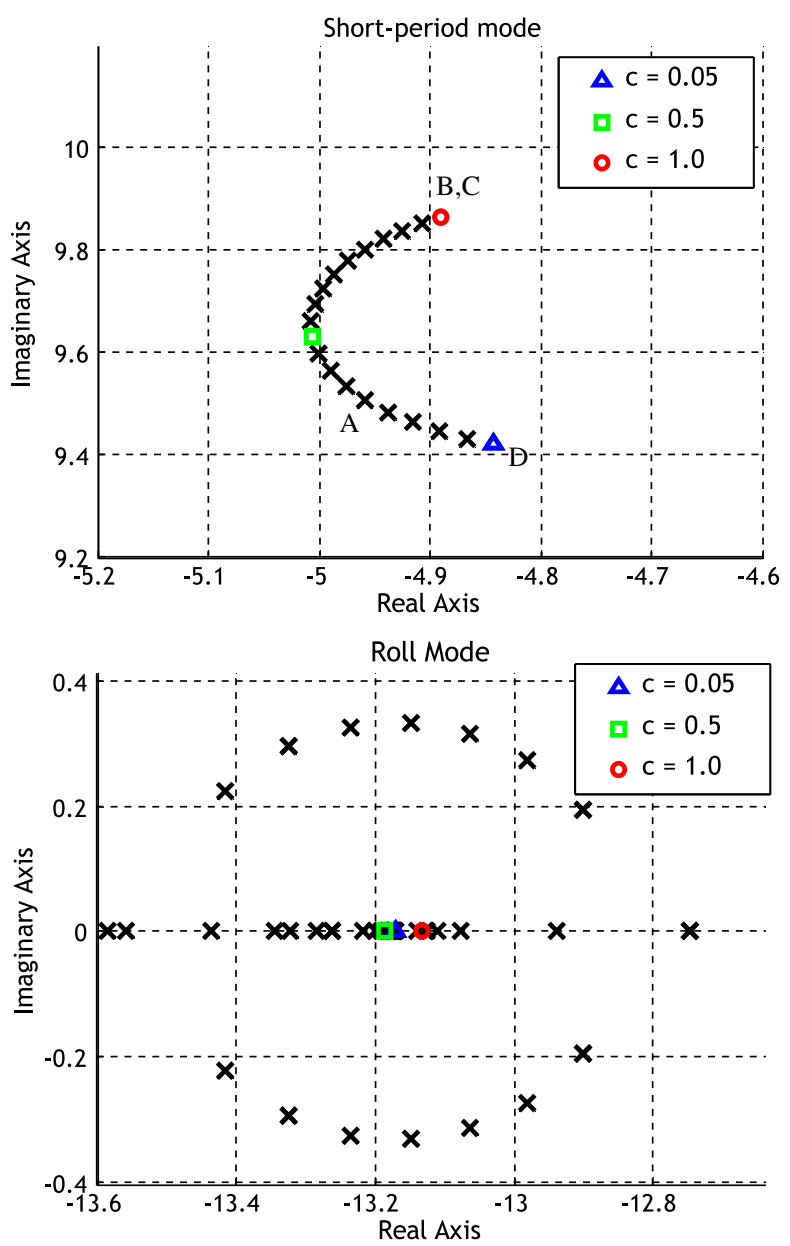

Fig. 9 Damping coefficient variation; A: maximum mode damping, B: minimum mode damping, $C$ : maximum natural frequency, $D$ : minimum natural frequency.

other mode affected is the roll mode, which has an increase in time constant of $3.33 \%$ due to the mode mixing. These changes are indeed small, so the damping coefficient mainly affects the high-frequency flap behavior, not the low-frequency flight dynamic modes. Additionally, the changes in the stability derivatives are insignificant. The small changes in the modes due to the hinge damping are likely due to effects of the flap rate and acceleration, which are not predicted using a static analysis. This correlates with the eigenvectors of the mode, which have significantly larger components of flap rate than flap angle.

\section{Effect of Vertical Hinge Location on Fuselage}

The point of attachment of the wing root to the fuselage is varied vertically from the bottom of the $0.06 \mathrm{~m}$ diameter fuselage to the top in increments of $0.002 \mathrm{~m}$. The attachment point is at the center of the fuselage in all cases. The wing attachment point is a type of basic aircraft design parameter that can be used by engineers to tailor the dynamic mode characteristics and handling qualities. This is because the distance of the wing aerodynamic center to the c.g. of the vehicle will affect the sign and magnitude of the aerodynamic moments produced. As shown in Table 1, the vertical hinge location affects all of the low-order dynamic aircraft modes, except for the spiral mode, most notably affecting the longitudinal modes. As the vertical hinge location is moved, the pitching moment from the drag force changes.

While an aircraft designer may use wing attachment location and other basic configuration parameters as a means to tune the dynamics and handling qualities of an aircraft, Fig. 10 shows how a compliant hinge can greatly widen the range of possible mode characteristics.
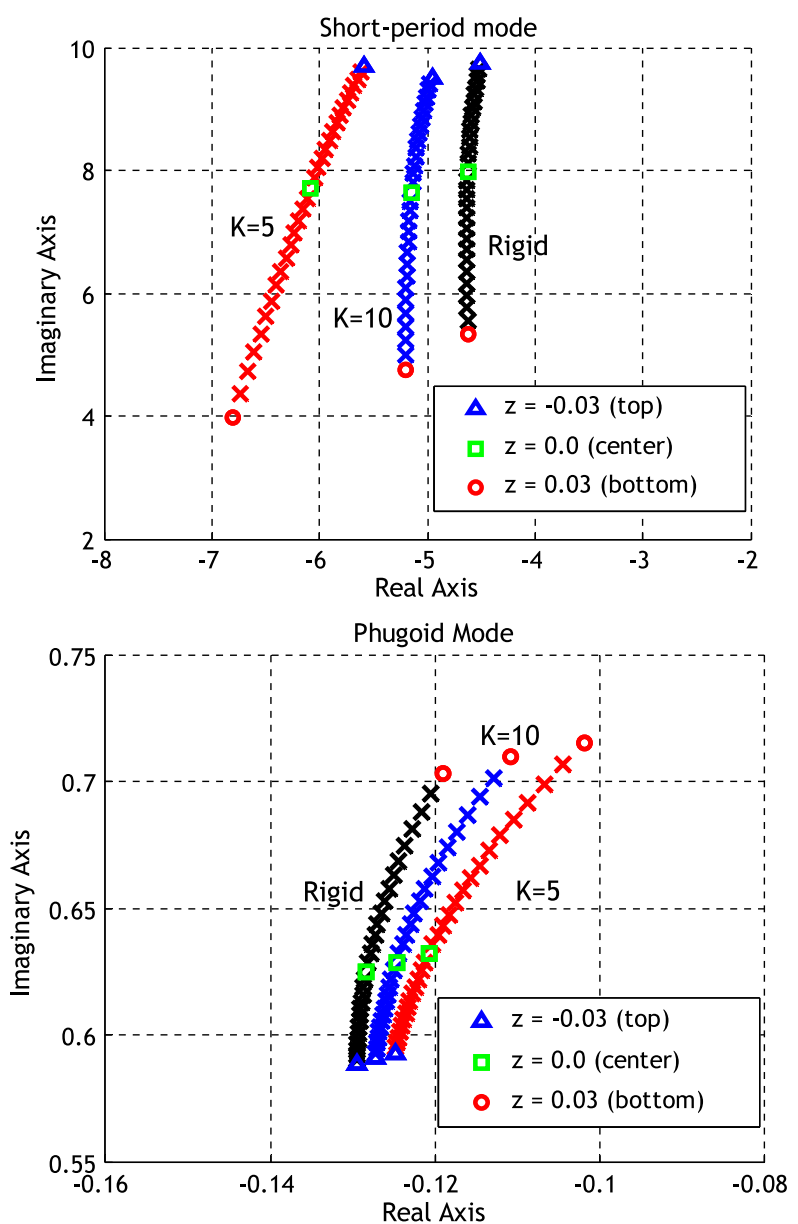

Fig. 10 Root loci for varying vertical hinge location at several springstiffness values.

Figure 10 compares the changes in the longitudinal modes as the vertical hinge location is varied for several spring stiffness values (5 and $10 \mathrm{~N} \cdot \mathrm{m} / \mathrm{rad}$, and rigid). The configuration alone or the presence of compliant hinge alone can be used to tailor the stability characteristics and handling qualities of the vehicle; however, the hinge location and hinge elasticity combined provide a way to significantly widen the range of achievable values. In the short-period mode shown in Fig. 10, varying the hinge location alone for a rigid aircraft provides a range of mode damping constants from 0.42 to 0.655 (top to bottom), which is a $55.8 \%$ increase in damping. The natural frequency varies from 10.7 to 7.06 , corresponding to a $34.3 \%$ decrease in the modal natural frequency. In comparison, a very soft spring increases the damping from 0.499 to 0.864 , corresponding to a $73.1 \%$ increase, which adds significantly more modal damping than the rigid aircraft. The change in natural frequency is fairly similar to the rigid case, decreasing from 11.2 to 7.88 , a change of $30 \%$. In the phugoid mode, Fig. 10, changing only the vertical hinge location (rigid case), the mode damping decreases from 0.2150 to 0.167 , a $22.3 \%$ decrease. With a very soft spring, the mode damping decreases from 0.206 to 0.141 , corresponding to a $31.6 \%$ decrease in damping. Thus, a very soft spring provides a significant increase in the range of the mode. The variation in natural frequency between the elastic and rigid cases is less apparent for both longitudinal modes. It is of note that, when the damping of the short-period mode increases, the damping in the phugoid decreases, so there are competing values.

For the nominal configuration with a spring stiffness of $10 \mathrm{~N}$. $\mathrm{m} / \mathrm{rad}$ presented in Table 1 , the combination of compliant hinge and variation in the vertical hinge location produces a $52 \%$ change in natural frequency of the short-period mode from minimum to maximum. This is a $131 \%$ change in the CAP of the aircraft. The damping coefficient can be increased by $60 \%$. These numbers mean a 
marginally Level 3 or Level 4 rated aircraft could become a Level 1 rated aircraft. While the damping of the phugoid degrades to obtain these improvements in the short period, the phugoid damping is still above the Level 1 minimum damping criteria for the phugoid.

In the lateral modes, the Dutch-roll damping ratio changes by $4.6 \%$ with a minute change in natural frequency, increasing as the hinge is moved from the bottom to the top. The flying-qualities criteria for the Dutch-roll mode consist of minimum values for the damping, natural frequency, and the multiplication of the damping ratio and natural frequency. In this study, the damping ratio multiplied by the natural frequency changed by $4.17 \%$. The roll-mode time constant only changes by $1.7 \%$; the maximum occurs with the hinge located at the top of the fuselage, and the minimum occurs when the wing is hinged at the center of the fuselage. The effect on the lateral modes is minor.

\section{Effect of Spanwise Hinge Location on Wing}

Rather than hinging the wing at its root where it connects to the fuselage, the hinge is moved to various locations along the span of the wing, such that a smaller portion of the wing can flap. This spanwise location is depicted in Fig. $\underline{5 b}$ by the distance $e$. The span of each wing is $0.4 \mathrm{~m}$, and the hinge location is varied from root to tip in increments of $0.05 \mathrm{~m}$. The results are listed in Table 1 and presented in Fig. 11. It is expected that moving the hinge out to the wingtip will increase the rigidity of the aircraft, so the trends in modal changes should be similar to those of stiffening the spring. In the phugoid mode, the damping of the mode changes by $2.2 \%$, increasing as the hinge is moved from root to tip. The short-period-mode damping ratio decreases $9 \%$ as the hinge location varies from root to tip. The changes in the natural frequencies of both longitudinal modes are negligible. The spanwise hinge location also has a trivial effect on the lateral modes of the vehicle. This closely follows the modal changes seen in the spring-stiffness results when the stiffness is increased from 10 to $75 \mathrm{~N} \cdot \mathrm{m} / \mathrm{rad}$. These results suggest that the hinge location could be moved slightly for ease of fabrication, or to avoid control surfaces, propulsive devices, inner ribs, etc., on the wing without significantly impacting the handling qualities of the aircraft.

\section{Effect of $\delta_{3}$ Hinge Orientation}

Implementation of a compliant hinge in the wing structure allows pitch-flap coupling to be introduced to produce wash-in or wash-out, which can have profound implications on the lower-order dynamic modes of the vehicle, as seen in the results presented in Table 1 and Fig. 12. The $\delta_{3}$ hinge orientation is varied from -90 to +90 deg by increments of $1 \mathrm{deg}$. The left-wing and right-wing $\delta_{3}$ angles are always equal and opposite so as to produce the same pitch-flap coupling on either wing. For example, when the left wing has a $+\delta_{3}$ angle and the right wing a negative $\delta_{3}$ angle, a flap up, or increase in

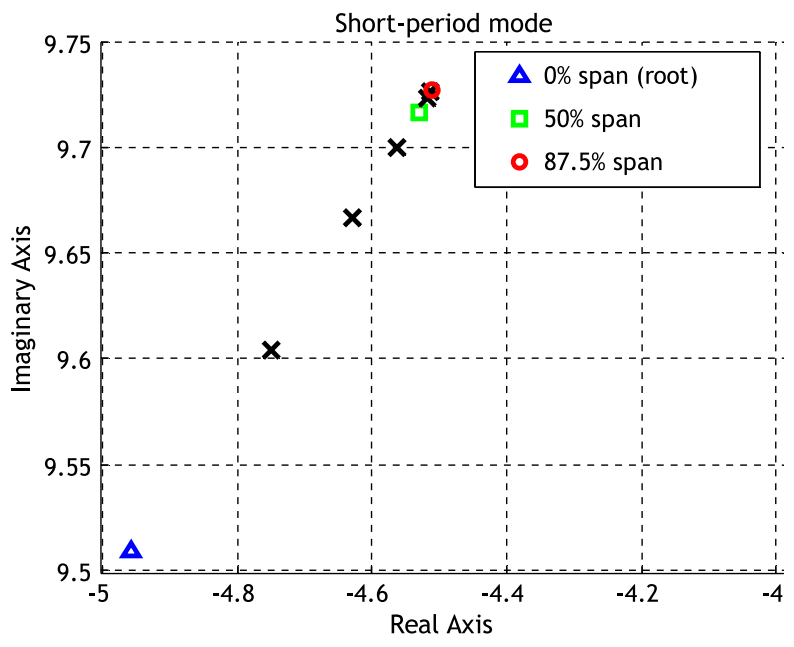

Fig. 11 Changes in dynamic mode as spanwise hinge location is varied. dihedral, of both wings will produce an increase in the angle of attack of both wings.

As seen in Table 1 and Fig. 12 , the $\delta_{3}$ orientation of a compliant hinge can affect all of the low-order modes. The short-period mode has the lowest damping when the left hinge has -45 deg hinge orientation and the right $+45 \mathrm{deg}$, and becomes overdamped when the left hinge has $+45 \mathrm{deg}$ and right wing $-45 \mathrm{deg}$ angle. The mode is overdamped the most at an angle of $+48 \mathrm{deg}$ on the left wing, and $-48 \mathrm{deg}$ on the right wing. The opposite is true for the phugoid mode, however. In that mode, the lowest damping occurs when the left hinge is at a $+45 \mathrm{deg}$ angle, right hinge at $-45 \mathrm{deg}$, and the highest damping occurs when the left hinge is at $-45 \mathrm{deg}$ and right hinge at $+45 \operatorname{deg} \delta_{3}$ orientation. Figure 12 is annotated with the hinge orientations for clarification. The short-period damping is higher with positive left-hinge orientation and negative right-hinge orientation because, for example, if there is a negative pitch-rate disturbance, this will increase the angle of attack on the wings. This angle-of-attack increase will cause the wings to flap up, further increasing the angle attack and lift, and thereby producing a positive pitching moment that will oppose the negative pitch disturbance.

The $\delta_{3}$ orientation has the largest effect on the roll mode of any parameter studied. The minimum time to half occurs with a $\delta_{3}$ angle of $+67 \mathrm{deg}$ on the left wing and $-67 \mathrm{deg}$ angle on the right wing. In this configuration, when a positive roll-rate disturbance occurs, the relative velocity will increase the angle of attack on the right wing and decrease the angle of attack on the left wing. This will increase the lift on the right wing and decrease the lift on the left wing, causing a flap up on the right wing and a flap down on the left. The differential flap causes differential angle-of-attack changes on the wing, producing a negative roll moment to oppose and damp out the initial positive rollrate disturbance.

The hinge orientation significantly impacts the flying-quality criteria of the longitudinal modes. The natural frequency of the shortperiod mode changes $37 \%$, thereby changing the CAP $87.5 \%$. The damping ratio increases $217 \%$. However, the damping ratio decreases as the natural frequency and CAP increase. The hinge orientation can improve the flying qualities of the vehicle from one Level rating to another, or even from Level 3 to Level 1. The phugoid-mode natural frequency increases by $120 \%$. The range of damping ratios seen shows that the damping ratio of the mode could be increased $369 \%$.

In the lateral modes, the Dutch-roll damping is not significantly impacted by hinge orientation, but does change $1.9 \%$. The minimum Dutch-roll damping occurs in the $+45 \mathrm{deg}$ left-wing $\delta_{3}$ orientation, -45 deg right-wing $\delta_{3}$ orientation configuration, and the maximum damping in the Dutch-roll mode occurs in the opposite configuration with $-45 \mathrm{deg}$ left wing $\delta_{3}$ and $+45 \mathrm{deg} \delta_{3}$ right wing. The rollmode time constant changes $6 \%$, and the spiral mode time to double changes $4.3 \%$.

Significant changes in the stability derivatives are noted as the $\delta_{3}$ angle is varied, which drive the dynamic mode changes. In the shortperiod mode, $M_{w}$ is most negative at $-45 \mathrm{deg}$ left-wing angle and +45 deg right-wing angle, corresponding to maximum natural frequency, and is least negative at a $+45 \mathrm{deg}$ left-wing, $-45 \mathrm{deg}$ right-wing orientation. The stability derivative $Z_{w}$ becomes less negative with a -45 deg left-wing angle and +45 deg right-wing angle, which, combined with the increase in natural frequency for this configuration, reduced the short-period-mode damping. The maximum damping occurs at the $+45 \mathrm{deg}$ left-wing, $-45 \mathrm{deg}$ right-wing orientation, where $Z_{w}$ is $23 \%$ more negative than the nominal configuration with zero $\delta_{3}$ orientation. These changes in $Z_{w}$ correspond to the changes in $M_{w}$ because the c.g. of the vehicle is behind the aerodynamic center of the main wing. As the vehicle is perturbed in $w$, the wings flap, producing a change in the angle of attack for nonzero $\delta_{3}$ angles, which in turn affects the wing aerodynamic forces and moments. In the $+45 \mathrm{deg}$ left-wing, -45 deg right-wing case, a positive $w$ perturbation would flap up the wings, increasing the angle of attack and lift, providing a restoring force that damps out the short-period mode, hence the increase in damping in this case. In the -45 deg left-wing, +45 deg right-wing case, a positive $w$ perturbation flaps up the wings, but decreases the angle of attack and lift, reducing the damping in the short-period 

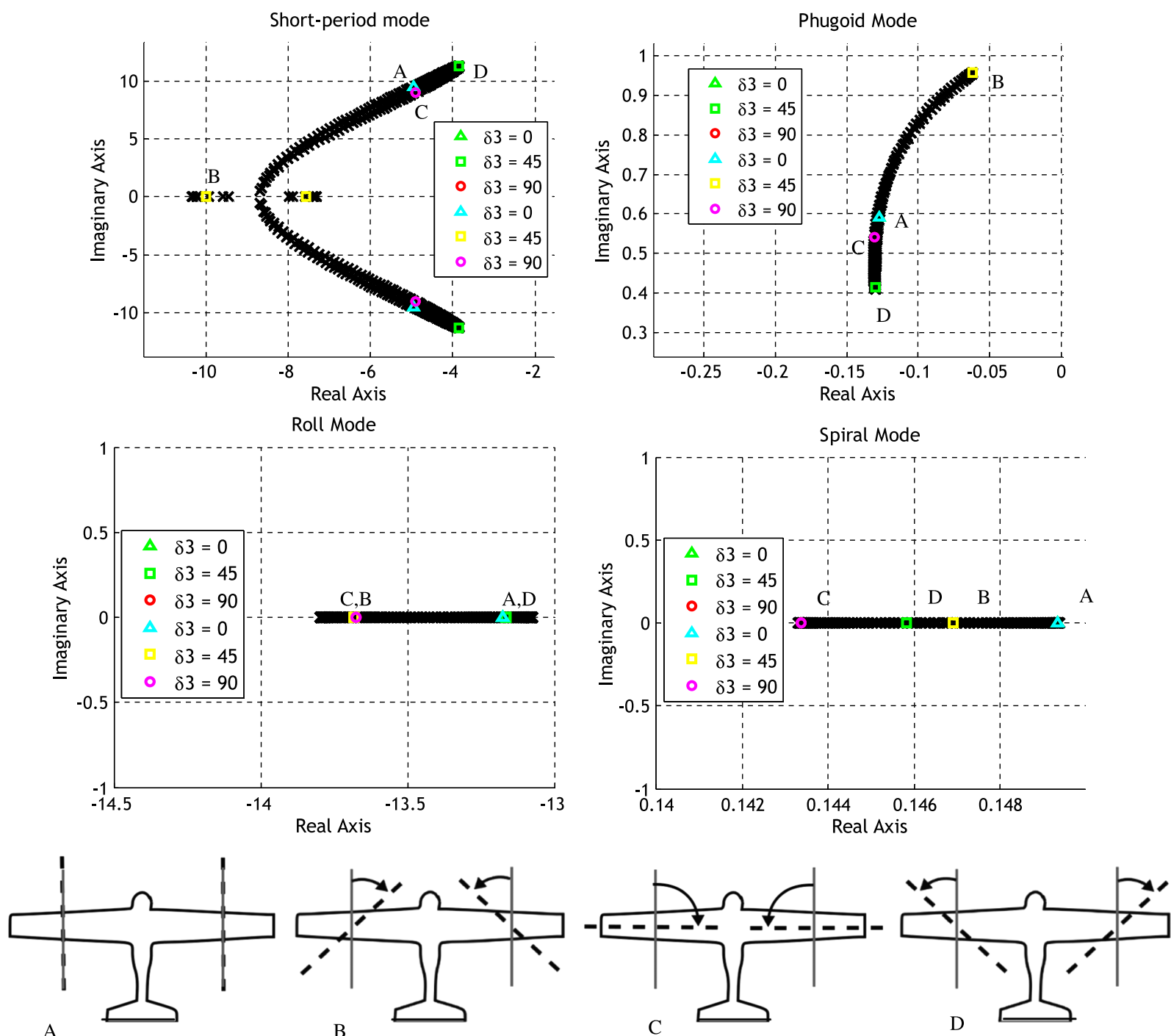

Fig. 12 Changes in dynamic modes with changes in $\delta_{3}$ hinge orientations.

mode. The pitch-damping derivative $M_{q}$ also contributes to the damping changes, although much less so than the derivatives with respect to $w$. Original expectations were that $\delta_{3}$ angles of $90 \mathrm{deg}$ on each wing would produce the most damping, because the wing flapping would directly correspond to an angle-of-attack change and the flap angles would be larger because rotation occurs about the wing body axis of lowest inertia. Several terms factor into the effect of flexibility on the $Z_{w}$ stability derivative in the static aeroelastic analysis. The derivatives of $Z$ aerodynamic force with respect to flap angle $\beta_{L}$ and $\beta_{R}$, as well as the derivatives of $\ddot{\beta}_{L}$ and $\ddot{\beta}_{R}$ with respect to $w$, are much larger for the $90 \mathrm{deg} \delta_{3}$ case. However, the coupling between the flap angle of the opposite wing on the flap rate of a wing (e.g., the derivative $\partial \ddot{\beta}_{L} / \partial \beta_{R}$ ) is significantly larger for the $45 \mathrm{deg} \delta_{3}$ case, causing it to have the most negative $Z_{w}$ derivative, and thereby maximum damping.

In the phugoid mode, the natural frequency is largely dependent on the aerodynamic stability derivative $Z_{u}$. In the +45 deg left-wing, -45 deg right-wing case, this derivative decreases, becoming more negative, by $21.9 \%$, which increases the magnitude of the derivative and thereby increases the natural frequency. The derivative $X_{u}$ affects the damping of the mode and becomes more positive, which reduces the magnitude, by $8.26 \%$. This reduction in the $X_{u}$ derivative combined with the increase in the natural frequency reduces the damping of the mode in this case from the nominal configuration with no $\delta_{3}$. In the opposite case with -45 deg left-wing, +45 deg right-wing $\delta_{3}$ angles, $Z_{u}$ becomes more positive, which decreases the magnitude of the derivative and natural frequency of the phugoid mode. The changes in stability derivatives are related to the coupling of the angle of attack with the flap angle for nonzero $\delta_{3}$ angles. The derivatives of the aerodynamic forces $X$ and $Z$ with respect to flap angles make the largest contribution to the changes in $X_{u}$ and $Z_{u}$ calculated. These changes in stability derivatives correspond to the changes in the natural frequency and damping, and thereby handling qualities, of the mode.

The roll mode depends mostly on the roll-damping stability derivative $L_{p}$ and the $N_{p}$ derivative terms. Changes in $N_{p}$ with varying $\delta_{3}$ angles are insignificant, but the roll-damping term $L_{p}$ is more negative, thereby providing more damping and reducing the time to half of the roll mode, at $\delta_{3}$ hinge orientations of $+65 \mathrm{deg}$ on the left-wing and $-65 \mathrm{deg}$ on the right-wing case. At orientations of -22 deg left-wing, +22 deg right-wing $\delta_{3}$ angles, a reduction in the magnitude of roll damping $L_{p}$ is noted from the nominal case, corresponding to the maximum time to half of the mode noted in Table 1 . This corresponds to changes in the mode noted in the root locus. The spiral mode is mostly affected by the aerodynamic stability derivatives for yaw damping $N_{r}$, weathercock stability $N_{v}$, dihedral stability $L_{v}$, and roll due to yaw coupling $L_{r}$. Weathercock stability is destabilizing, and dihedral stability is stabilizing for the spiral mode. Comparing the cases of zero $\delta_{3}$ angle with $90 \mathrm{deg} \delta_{3}$ angle, which have the maximum and minimum time to double of the spiral mode, the 90 deg case has a larger (more negative) dihedral stability derivative $L_{v}$ and also has a slightly smaller roll-yaw coupling term $L_{r}$. Both of these stabilize the spiral mode and reduce the time to double of the mode for the $90 \operatorname{deg} \delta_{3}$ configuration. 


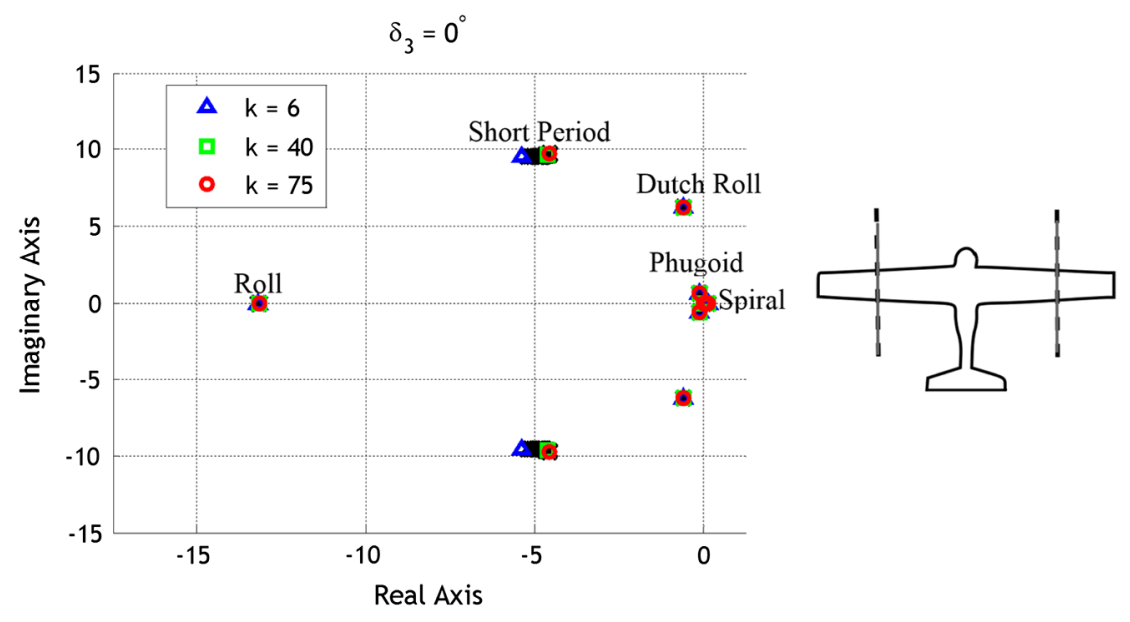

a)

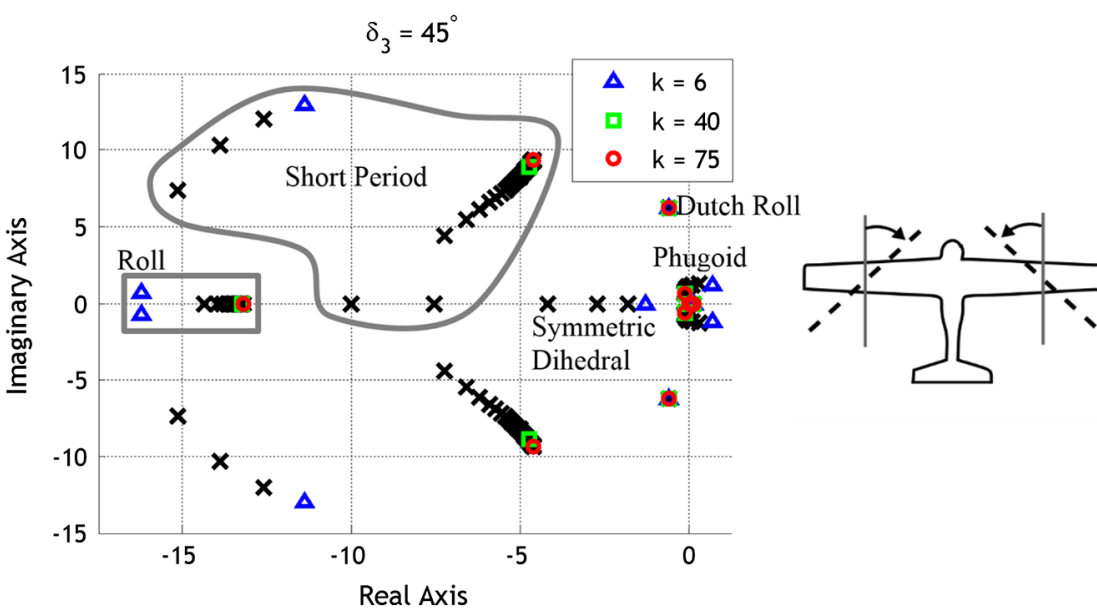

b)

Fig. 13 Root loci for varying spring stiffness for a) $\delta_{3}$ of $0 \mathrm{deg}$, and b) $\delta_{3}$ of $+45 \mathrm{deg}$ on left wing, -45 deg on right wing.

The flight dynamic modes of aircraft configurations with nonzero $\delta_{3}$ angles exhibit an increased sensitivity to other hinge design parameters, such as spring stiffness and damping. This coupling between the parameters significantly extends the range of achievable mode tailoring. For example, in Fig. 13a, the spring stiffness is varied from 6 to $75 \mathrm{~N} \cdot \mathrm{m} / \mathrm{rad}$ in increments of $1 \mathrm{~N} \cdot \mathrm{m} / \mathrm{rad}$ with the $\delta_{3}$ angle set at the nominal configuration of $0 \mathrm{deg}$. Minor movement in the short-period mode due to variations in the spring stiffness is observed. In comparison, Fig. 13b shows the effect of the same variation in spring stiffness on an aircraft with the hinge $\delta_{3}$ angle oriented at $+45 \mathrm{deg}$ on the left wing and $-45 \mathrm{deg}$ on the right wing. The higher spring-stiffness values approach a rigid aircraft configuration, so the modal values match between Figs. 13a and $13 \mathrm{~b}$ in those cases. However, as the hinge becomes more flexible, significantly more movement is apparent in the modes of the $45 \mathrm{deg}$ $\delta_{3}$ hinge case, Fig. $13 \mathrm{~b}$. The phugoid mode crosses the imaginary axis and is unstable for spring-stiffness values less than $9 \mathrm{~N} \cdot \mathrm{m} / \mathrm{rad}$. The short-period mode increases damping until it becomes an overdamped, real mode at a spring stiffness of $10 \mathrm{~N} \cdot \mathrm{m} / \mathrm{rad}$, but then breaks out into an oscillatory mode at a much higher natural frequency as the stiffness is further reduced. The roll mode also experiences a notable change in time to half, unlike the nominal case. With a nonzero $\delta_{3}$ angle, the angle of attack changes as the wing flaps. This introduces coupling between the lateral and longitudinal motions, which greatly impacts the flight dynamic modes as more flexibility is introduced.

\section{Effect of $\delta_{2}$ Hinge Orientation}

The $\delta_{2}$ hinge orientation, depicted in Fig. $\underline{5 \mathrm{c}}$, is varied from -90 to $+90 \mathrm{deg}$ by increments of $1 \mathrm{deg}$. The $\delta_{2}$ hinge orientation produces sweep-flap coupling. The left-wing and right-wing $\delta_{2}$ angles are always equal for symmetry. With a $+\delta_{2}$ orientation, a flap up, increasing the dihedral, will also sweep the wings back. For a $-\delta_{2}$ orientation, a flap up will sweep the wings forward. As reported in Table 1 and Fig. 14, this can significantly change the longitudinal modes of the vehicle, as well as affect the damping of the Dutch-roll mode. The phugoid mode and the short-period mode show opposite trends in terms. In the short period, the lowest damping occurs at $+45 \mathrm{deg} \delta_{2}$ angles, and the highest occurs at $-45 \operatorname{deg} \delta_{2}$ angles. In the Dutch-roll mode, the highest mode damping occurs at $90 \mathrm{deg}$ hinge angles, whereas the lowest damping is without any $\delta_{2}$ angle present.

The damping ratio of the Dutch-roll mode changes $1.6 \%$. However, the $\delta_{2}$ hinge orientation causes large variations in the response of the longitudinal modes. The natural frequency of the short-period mode changes $28.7 \%$, corresponding to a $65.7 \%$ increase in the CAP. The maximum change in the damping ratio of the short-period mode due to the $\delta_{2}$ hinge orientation is $88 \%$. This, too, provides a means to improve the Level rating of the flying qualities of the aircraft. The phugoid mode experiences possible increases in the natural frequency of $50.2 \%$ and in the damping ratio of $71.5 \%$.

Employing static aeroelastic analysis, changes in the short-periodmode natural frequency are largely due to changes in the stability derivative $M_{w}$. Interestingly, the derivative $Z_{w}$ does not change significantly. The changes in $M_{w}$ and short-period mode are likely due to the movement in the aerodynamic center of the wing relative to the c.g. of the vehicle as the wing sweeps forward or aft with flapping. The pitch-damping derivative $M_{q}$, which also contributes to the damping of the short-period mode, changes slightly by $2.4 \%$ from the nominal case of zero $\delta_{2}$ angle to $-45 \operatorname{deg} \delta_{2}$ angles to increase the 

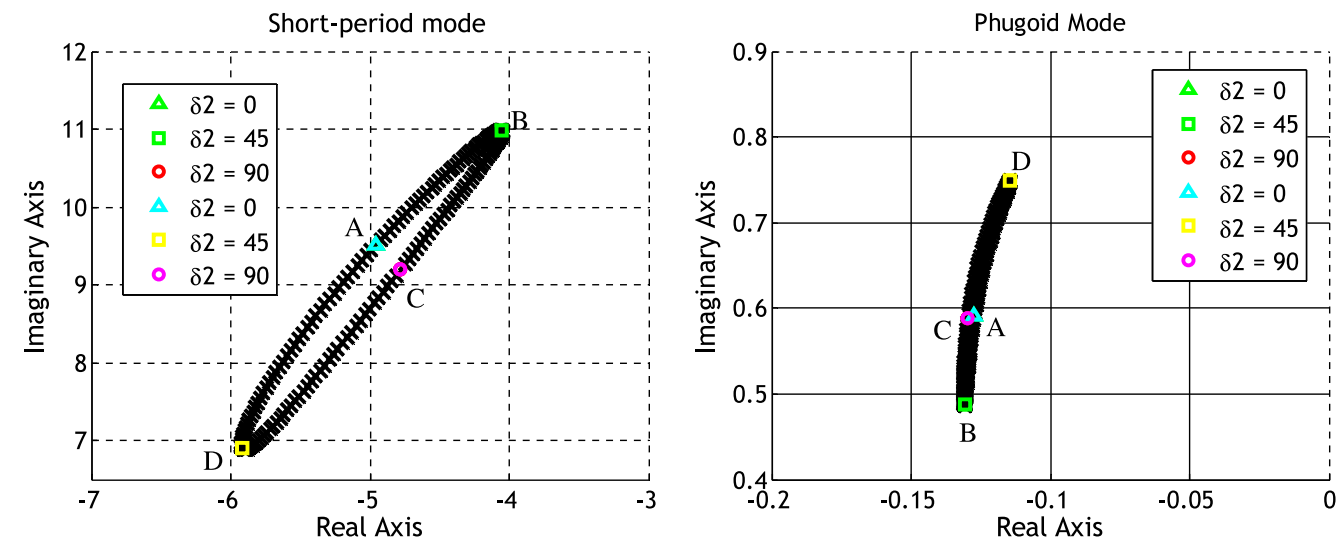

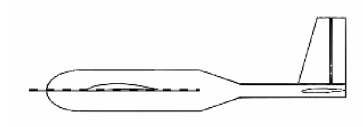

A

B

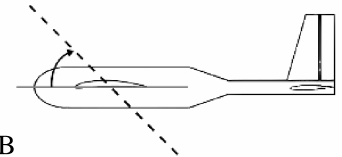

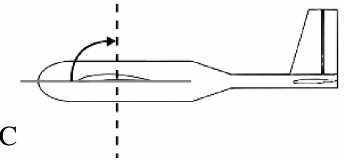

$\mathrm{D}$

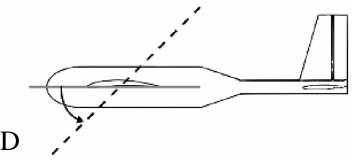

Fig. 14 Changes in dynamic modes with changes in $\delta_{2}$ hinge orientations.

damping. In the phugoid, the derivative $Z_{u}$ does not change significantly with varying $\delta_{2}$ angle. The derivative $X_{u}$, which appears in the damping of the phugoid, changes slightly by $2.7 \%$ from the nominal case of zero $\delta_{2}$ angle to $-45 \operatorname{deg} \delta_{2}$ angles, reducing the mode damping. These small changes do not explain the large changes in the phugoid mode observed from the root locus with varying $\delta_{2}$ hinge orientations, suggesting that the flapping velocity and acceleration terms, which are not accounted for in the static aeroelastic analysis, are important to the phugoid-mode characteristics.

\section{Conclusions}

The emergence of cost-effective multimaterial manufacturing methods allows complex structures to be fabricated that incorporate flexible structures, which act as discrete elastic hinges into an otherwise rigid structure. This presents new possibilities for designing aircraft, particularly small unmanned aircraft. Rather than using typical design parameters, such as the geometry and arrangement of the aircraft components, the lifting surfaces can be constructed with a chordwise strip of relatively soft structural material, which functions as an elastic discrete hinge. The characteristics of the hinge can then be used to tailor the dynamics of the vehicle.

From a series of parametric trade studies, it is shown that an elastic hinge embedded in the wing causes coupling between the flexible modes and traditional low-order dynamic modes, changing the flight dynamic response of the vehicle. Along with the stiffness of the joint material, the orientation of the hinge introduces coupling of the lateral and longitudinal motions of the wing to affect the response. Together, these provide a range of short-period-mode characteristics from Level 4 to Level 1 flying-qualities ratings, such that the elasticity and hinge orientation can be used to tailor the shortperiod handling qualities. Nonzero hinge orientations also show a significant increase in sensitivity to variations in the stiffness and damping of the hinge, which allows even further tailoring of the modes. Static aeroelastic analysis highlights the changes in aerodynamic stability derivatives that drive the changes in the modes with variations in the hinge parameters, and identifies cases in which the modal changes are largely affected by the transient dynamics, for which a static analysis is insufficient to explain the results. While previous work generally focused on highly flexible wings, which degraded flying qualities, this paper presents a full sweep through a wide range of stiffness values and shows how decreasing the stiffness can improve handling qualities, particularly in the longitudinal modes. It is also shown how the introduction of a discrete structural hinge can increase the range of short-period response characteristics produced by configuration changes of the vehicle alone. The lateral modes of the vehicle are not significantly impacted by the presence of the compliant hinge. The reasons for this could stem from the dihedral being kept fixed at a certain value. The lateral modes also depend on the aerodynamics of the vertical tail, for which addition of compliancy was not examined in this paper.

\section{Acknowledgment}

This material is based upon the work supported by the National Science Foundation Graduate Research Fellowship Program under grant number DGE-1148903.

\section{References}

[1] Dimitrov, D., Schreve, K., and de Beer, N., "Advances in Three Dimensional Printing - State of the Art and Future Perspectives," Rapid Prototyping Journal, Vol. 12, No. 3, 2006, pp. 136-147. doi: $10.1108 / 13552540610670717$

[2] Gouker, R., Gupta, S., Bruck, H., and Holzschuh, T., "Manufacturing of Multi-Material Compliant Mechanisms Using Multi-Material Molding," International Journal of Advanced Manufacturing Technology, Vol. 30, Nos. 11-12, 2006, pp. 1049-1075. doi:10.1007/s00170-005-0152-4

[3] Choi, J., Kim, H., and Wicker, R., "Multi-Material Stereolithography," Journal of Materials Processing Technology, Vol. 211, No. 3, 2011, pp. 318-328. doi:10.1016/j.jmatprotec.2010.10.003

[4] Cutkosky, M. R., and Kim, S., "Design and Fabrication of MultiMaterial Structures for Bioinspired Robots," Philosophical Transactions of the Royal Society of London, Series A: Mathematical, Physical and Engineering Sciences, Vol. 367, No. 1894, 2009, pp. 1799-1813. doi:10.1098/rsta.2009.0013

[5] Bejgerowski, W., Gerdes, J. W., Gupta, S. K., and Bruck, H. A., "Design and Fabrication of Miniature Compliant Hinges for MultiMaterial Compliant Mechanisms," International Journal of Advanced Manufacturing Technology, Vol. 57, Nos. 5-8, 2011, pp. 437-452.

[6] Pankonien, A., and Inman, D. J., "Experimental Testing of Spanwise Morphing Trailing Edge Concept," SPIE Conference on Smart Structures and Materials: Active and Passive Smart Structures and Integrated Systems, Vol. 8688, Soc. of Photo-Optical Instrumentation Engineers, Bellingham, WA, 2013, pp. 1-13. doi: $10.1117 / 12.2009400$

[7] Webb, A., and Costello, M., "Wing Articulation of Micro Air Vehicles to Reduce Gust Sensitivity," AIAA Atmospheric Flight Mechanics Conference, AIAA Paper 2008-6712, Aug. 2008.

[8] Oduyela, A., and Slegers, N., "Gust Mitigation of Micro Air Vehicles Using Passive Articulated Wings," Scientific World Journal, Vol. 2014, 2014, pp. 1-10. doi: $10.1155 / 2014 / 598523$ 
[9] Porter, R. F., and Brown, J. H., Jr., "Evaluation of the Gust-Alleviation Characteristics and Handling Qualities of a Free-Wing Aircraft," NASA CR-1523, April 1970.

[10] Tanaka, H., Whitney, J. P., and Wood, R. J., "Effect of Flexural and Torsional Wing Flexibility on Lift Generation in Hoverfly Flight," Integrative and Comparative Biology, Vol. 51, No. 1, 2011, pp. $142-150$. doi:10.1093/icb/icr051

[11] Krus, P., "Natural Methods for Flight Stability in Birds," World Aviation Congress, AIAA Paper 1997-5653, 1997, pp. 1-6.

[12] Leishman, J. G., Principles of Helicopter Aerodynamics, 2nd ed., Cambridge Univ. Press, New York, 2006, pp. 198-199.

[13] Waterman, W. D., Santa Monica Canyon, CA, U.S. Patent Application for "Aeroplane Control," Serial No. 312,242, filed 13 Oct. 1928, Dec. 1930.

[14] Shirk, M. H., Hertz, T. J., and Weisshaar, T. A., "Aeroelastic TailoringTheory, Practice, and Promise," Journal of Aircraft, Vol. 23, No. 1, 1986, pp. 6-18. doi: $10.2514 / 3.45260$

[15] Weisshaar, T. A., Nam, C., and Batista-Rodriguez, A., "Aeroelastic Tailoring for Improved UAV Performance," 39th AIAA/ASME/ASCE/ AHS/ASC Structures, Structural Dynamics, and Materials Conference and Exhibit, AIAA, Reston, VA, 1998, pp. 1-13.

[16] Pitt, D., "Static and Dynamic Aeroelastic Analysis of Structural Wing Fold Hinges That Are Employed as an Aeroelastic Tailoring Tool," 45th AIAA/ASME/ASCE/AHS/ASC Structures, Structural Dynamics, and Materials Conference, AIAA, Reston, VA, April 2004, pp. 1-11.

[17] Ameri, N., Lowenberg, M. H., and Friswell, M. I., "Modelling the Dynamic Response of a Morphing Wing with Active Winglets," AIAA Atmospheric Flight Mechanics Conference, AIAA Paper 2007-6500, Aug. 2007.

[18] Abdulrahim, M., and Lind, R., "Flight Testing and Response Characteristics of a Variable Gull-Wing Morphing Aircraft," AIAA Guidance, Navigation, and Control Conference, AIAA Paper 20045113, Aug. 2004.

[19] Abdulrahim, M., and Lind, R., "Control and Simulation of a Multi-Role Morphing Micro Air Vehicle," AIAA Guidance, Navigation, and Control Conference, AIAA Paper 2005-6481, Aug. 2005.

[20] Abdulrahim, M., and Lind, R., "Using Avian Morphology to Enhance Aircraft Maneuverability," AIAA Atmospheric Flight Mechanics Conference, AIAA Paper 2006-6643, Aug. 2006.

[21] Agenbag, D. S., Theron, N. J., and Huyssen, R. J., "Pitch Handling Qualities Investigation of the Tailless Gull-Wing Configuration," Journal of Aircraft, Vol. 46, No. 2, 2009, pp. 683-691. doi: 10.2514/1.39755

[22] Paranjape, A. A., Chung, S.-J., and Selig, M. S., "Flight Mechanics of a Tailless Articulated Wing Aircraft," Bioinspiration \& Biomimetics, Vol. 6, No. 2, 2011, Paper 026005. doi:10.1088/1748-3182/6/2/026005

[23] Paranjape, A. A., Chung, S.-J., Hilton, H. H., and Chakravarthy, A., "Dynamics and Performance of Tailless Micro Aerial Vehicle with Flexible Articulated Wings," AIAA Journal, Vol. 50, No. 5, 2012, pp. 1177-1188. doi:10.2514/1.J051447
[24] Babcock, J., and Lind, R., "Aeroelastic Effects of Wing Stiffness on the Flight Dynamics of a MAV," AIAA Atmospheric Flight Mechanics Conference, AIAA, Reston, VA, Aug. 2012, pp. 1-19.

[25] Waszak, M. R., and Schmidt, D. K., "Flight Dynamics of Aeroelastic Vehicles," Journal of Aircraft, Vol. 25, No. 6, June 1988, pp. 563-571. doi: $10.2514 / 3.45623$

[26] Weisshaar, T. A., and Zeiler, T. A., "Dynamic Stability of Flexible Forward Swept Wing Aircraft," AIAA Atmospheric Flight Mechanics Conference, AIAA, New York, Aug. 1982, pp. 1-11.

[27] Cerra, J. J., II, Calico, R. A., and Noll, T. E., "Modelling of Rigid-Body and Elastic Aircraft Dynamics for Flight Control Development," AIAA Astrodynamics Conference, AIAA, New York, 1986, pp. 386-395.

[28] Siepenkotter, N., and Alles, W., "Stability Analysis of the Nonlinear Dynamics of Flexible Aircraft," Aerospace Science and Technology, Vol. 9, No. 2, 2005, pp. 135-141. doi:10.1016/j.ast.2004.10.005

[29] Tuzcu, I., "On the Stability of Flexible Aircraft," Aerospace Science and Technology, Vol. 12, No. 5, 2008, pp. 376-384. doi:10.1016/j.ast.2007.09.003

[30] Silvestre, F. J., and Paglione, P., "Dynamics and Control of a Flexible Aircraft," AIAA Atmospheric Flight Mechanics Conference, AIAA, Reston, VA, Aug. 2008, pp. 1-18.

[31] Baghdadi, N., Lowenberg, M. H., and Isikveren, A. T., "Analysis of Flexible Aircraft Dynamics Using Bifurcation Methods," Journal of Guidance, Control, and Dynamics, Vol. 34, No. 3, 2011, pp. 795-809. doi: $10.2514 / 1.51468$

[32] Andrews, S. P., "Modelling and Simulation of Flexible Aircraft: Handling Qualities with Active Load Control," Ph.D. Dissertation, School of Engineering, Cranfield Univ., Cranfield, England, U.K., 2011.

[33] Newman, B., "Proposed Flying Quality Metrics and Simulation Studies for Elastic Vehicles," AIAA Atmospheric Flight Mechanics Conference, AIAA Paper 1996-3423, July 1996, pp. 500-509.

[34] Schmidt, D. K., and Raney, D. L., "Modeling and Simulation of Flexible Flight Vehicles," Journal of Guidance, Control, and Dynamics, Vol. 24, No. 3, May 2001, pp. 539-546. doi:10.2514/2.4744

[35] Phillips, W. F., and Snyder, D. O., "Modern Adaptation of Prandtl's Classic Lifting-Line Theory," Journal of Aircraft, Vol. 37, No. 4, 2000 , pp. 662-670. doi: $10.2514 / 2.2649$

[36] Anderson, J. D. Jr., Corda, S., and Van Wie, D. M., "Numerical Lifting Line Theory Applied to Drooped Leading-Edge Wings Below and Above Stall," Journal of Aircraft, Vol. 17, No. 12, 1980, pp. 898-904. doi: $10.2514 / 3.44690$

[37] Selig, M. S., "Modeling Full-Envelope Aerodynamics of Small UAVs in Realtime," AIAA Atmospheric Flight Mechanics Conference, AIAA Paper 2010-7635, Aug. 2010.

[38] Ogata, K., Modern Control Engineering, 4th ed., Prentice-Hall, Upper Saddle River, NJ, 2002, pp. 112-114.

[39] Anon., "Flying Qualities of Piloted Airplanes," MIL-SPEC, MIL-F8785C, 1980.

[40] Bisplinghoff, R. L., Ashley, H., and Halfman, R. L., Aeroelasticity, Dover, Mineola, NY, 1983, pp. 421-427. 